\title{
Stimulation Augments Spike Sequence Replay and Memory Consolidation during Slow-Wave Sleep
}

\author{
(ㄱina Wei, ${ }^{1}$ Giri P. Krishnan, ${ }^{1}{ }^{-}$Lisa Marshall, ${ }^{2,3}$ Thomas Martinetz, ${ }^{4}$ and Maxim Bazhenov ${ }^{1}$ \\ ${ }^{1}$ Department of Medicine, University of California, San Diego, La Jolla California 92093, ${ }^{2}$ Institute for Experimental and Clinical Pharmacology and \\ Toxicology, ${ }^{3}$ Center for Brain, Behavior and Metabolism, and ${ }^{4}$ Institute for Neuro- and Bioinformatics, University of Lübeck, 23562 Lübeck, Germany
}

\begin{abstract}
Newly acquired memory traces are spontaneously reactivated during slow-wave sleep (SWS), leading to the consolidation of recent memories. Empirical studies found that sensory stimulation during SWS can selectively enhance memory consolidation with the effect depending on the phase of stimulation. In this new study, we aimed to understand the mechanisms behind the role of sensory stimulation on memory consolidation using computational models implementing effects of neuromodulators to simulate transitions between awake and SWS sleep, and synaptic plasticity to allow the change of synaptic connections due to the training in awake or replay during sleep. We found that when closed-loop stimulation was applied during the Down states of sleep slow oscillation, particularly right before the transition from Down to Up state, it significantly affected the spatiotemporal pattern of the slow waves and maximized memory replay. In contrast, when the stimulation was presented during the Up states, it did not have a significant impact on the slow waves or memory performance after sleep. For multiple memories trained in awake, presenting stimulation cues associated with specific memory trace could selectively augment replay and enhance consolidation of that memory and interfere with consolidation of the others (particularly weak) memories. Our study proposes a synaptic-level mechanism of how memory consolidation is affected by sensory stimulation during sleep.
\end{abstract}

Key words: improve memory; memory consolidation; sleep; slow-wave oscillations; stimulation; targeted memory reactivation

Significance Statement

Stimulation, such as training-associated cues or auditory stimulation, during sleep can augment consolidation of the newly encoded memories. In this study, we used a computational model of the thalamocortical system to describe the mechanisms behind the role of stimulation in memory consolidation during slow-wave sleep. Our study suggests that stimulation preferentially strengthens memory traces when delivered at a specific phase of the slow oscillation, just before the Down to Up state transition when it makes the largest impact on the spatiotemporal pattern of sleep slow waves. In the presence of multiple memories, presenting sensory cues during sleep could selectively strengthen selected memories. Our study proposes a synaptic-level mechanism of how memory consolidation is affected by sensory stimulation during sleep.

\section{Introduction}

Memory formation depends on three general processes: encoding, consolidation, and retrieval. After new knowledge is en-

Received June 14, 2019; revised Oct. 14, 2019; accepted Nov. 3, 2019.

Author contributions: Y.W., G.P.K., and M.B. designed research; Y.W. performed research; Y.W. analyzed data; Y.W. wrote the first draft of the paper; Y.W., G.P.K., L.M., T.M., and M.B. edited the paper; Y.W., G.P.K., and M.B. wrote the paper.

This work was supported by National Science Foundation IIS-1724405, Defense Advance Research Projects Agency/Microsystems Technology Office Lifelong Learning Machines Program HR0011-18-2-0021, National Institutes of Health RF1MH117155, Office of Naval Research MURI N00014-16-1-2829, National Science Foundation/German Federal Ministry of Education and Research, Program US-German Collaboration in Neuroscience, under Grant Number (BMBF 01GQ1706).

The authors declare no competing financial interests.

Correspondence should be addressed to Maxim Bazhenov at mbazhenov@health.ucsd.edu.

https://doi.org/10.1523/JNEUROSCI.1427-19.2019

Copyright $\odot 2020$ the authors coded, it undergoes a phase of consolidation. Sleep facilitates this process by spontaneously reactivating the newly encoded memories (Walker and Stickgold, 2004; Diekelmann and Born, 2010; Born and Wilhelm, 2012; Rasch and Born, 2013). A common hypothesis is that the consolidation of memories during sleep occurs through the reactivation of the neuron ensembles engaged during learning (Euston et al., 2007; Ji and Wilson, 2007; Peyrache et al., 2009; Barnes and Wilson, 2014; Ramanathan et al., 2015).

The natural sleep cycle consists of several stages: Stage N1 (light), Stage N2, and Stage N3 (deep) of non-rapid eye movement (NREM) sleep and rapid eye movement (REM) sleep. During NREM sleep, sleep spindles, 7-14 Hz brief bursts of rhythmic waves, are the hallmark of N2 sleep (Loomis et al., 1935; Steriade et al., 1993; Andrillon et al., 2011), whereas slow oscillations (SO), characterized by repetitive $(<1 \mathrm{~Hz})$ transitions between $\mathrm{Up}$ 
and Down states in the cortical neurons, are mainly observed during N3 sleep (also referred to as slow-wave sleep [SWS]) (Blake and Gerard, 1937; Steriade et al., 1993, 2001). Presently, sensory stimulation during NREM sleep has been shown to modulate brain rhythms of sleep as well as affect memory performance across sleep. The sensory stimuli applied during sleep were either associated with the learning material in a training session before sleep, targeted memory reactivation (TMR) (Rasch et al., 2007; Rudoy et al., 2009; Antony et al., 2012; Cousins et al., 2014, 2016; Schönauer et al., 2014) or were unassociated to learning content (Ngo et al., 2013, 2015). In both cases, sleep stimulation could improve memory performance compared with the nonstimulation case (for review, see Oudiette and Paller, 2013; Schouten et al., 2017).

In TMR, auditory (Rudoy et al., 2009) or olfactory (Rasch et al., 2007) cues that were used during learning are presented again during NREM sleep, leading to an increase in memory performance after sleep. While many TMR studies use declarative memory tasks, TMR protocols may also enhance consolidation of hippocampus-independent procedural memories (Antony et al., 2012; Cousins et al., 2014, 2016; Schönauer et al., 2014). Antony et al. (2012) trained participants for two different motor sequence tasks with sensory cues and then presented the cue for one of the tasks during the nap. Following the nap, the cued task revealed higher performance than the uncued one, suggesting that TMR can enhance procedural memories. The amount of improvement correlated with the duration of SWS and the number of spindles during SWS (Antony et al., 2012). Similar improvements were found for the declarative component of a trained sequence (Cousins et al., 2014). Memory performance across a sleep period of $8 \mathrm{~h}$ without auditory cues was comparable with performance across a shorter sleep period of $3 \mathrm{~h}$ with cued activation (Schönauer et al., 2014). Auditory cues during SWS may not only enhance motor sequence performance but also increase the functional brain activity and connectivity in consolidation-relevant networks (Cousins et al., 2016). Interestingly, presentation of the cues during wakefulness was not effective for augmenting either declarative (Rasch et al., 2007) or procedural memories (Schönauer et al., 2014).

When the sensory stimuli were unassociated with the learning content, such as presenting pink noise during sleep, the amplitude of slow waves during sleep and retention performance were enhanced (Ngo et al., 2013, 2015). Stimulation was effective only when auditory "clicks" occurred in synchrony with the slow waves. In contrast, out-of-phase stimulation was ineffective (Ngo et al., 2013; Weigenand et al., 2016). Surprisingly, in a closed-loop auditory stimulation protocol, when more than two stimuli were presented sequentially at the adjacent cycles of the SO, it led to saturation and could not further enhance memory performance (Ngo et al., 2015).

In this new study, we used a biophysical model of the thalamocortical network to investigate the mechanisms behind the role of external stimulation (training-associated cues or sensory stimulation) on memory consolidation during SWS (N3 sleep). The model incorporated populations of thalamic and cortical neurons and implemented effects of neuromodulators to allow transitions between awake and SWS (Krishnan et al., 2016), as well as spike-timing-dependent plasticity (STDP) (Wei et al., 2016, 2018). Our study explains previous empirical data and provides insights into how synaptic reactivation within the thalamocortical network may be affected by sensory stimulation during sleep.
Table 1. Main parameters ${ }^{a}$

\begin{tabular}{|c|c|c|}
\hline Parameter & Value & Description \\
\hline$C_{m}$ & $1 \mu \mathrm{F} / \mathrm{cm}^{2}$ (TC; RE); $0.75 \mu \mathrm{F} / \mathrm{cm}^{2}$ (PY; IN) & Membrane capacitance \\
\hline \multicolumn{3}{|c|}{ Thalamic cells } \\
\hline S & $2.9 \times 10^{-4} \mathrm{~cm}^{2}(\mathrm{TC}) ; 1.43 \times 10^{-4} \mathrm{~cm}^{2}(\mathrm{RE})$ & Area of neurons \\
\hline$G_{L}$ & $0.01 \mathrm{mS} / \mathrm{cm}^{2}(\mathrm{TC}) ; 0.05 \mathrm{mS} / \mathrm{cm}^{2}$ (RE) & Leakage conductance \\
\hline$E_{L}$ & $-70 \mathrm{mV}(\mathrm{TC}) ;-77 \mathrm{mV}$ (RE) & Leakage reversal potential \\
\hline $\mathrm{G}_{\mathrm{KL}}$ & $0.024 \mathrm{mS} / \mathrm{cm}^{2}(\mathrm{TC}) ; 0.012 \mathrm{mS} / \mathrm{cm}^{2}$ (RE) & Potassium leakage conductance \\
\hline$E_{K}$ & $-95 \mathrm{mV}(\mathrm{TC} ; \mathrm{RE})$ & Potassium reversal potential \\
\hline$g_{k}$ & $10 \mathrm{mS} / \mathrm{cm}^{2}(\mathrm{RE}) ; 12 \mathrm{mS} / \mathrm{cm}^{2}(\mathrm{TC})$ & Maximal potassium conductance \\
\hline$g_{\mathrm{Na}}$ & $90 \mathrm{mS} / \mathrm{cm}^{2}$ (TC); $100 \mathrm{mS} / \mathrm{cm}^{2}$ (RE) & Maximal sodium conductance \\
\hline$g_{T}$ & $2.5 \mathrm{mS} / \mathrm{cm}^{2}$ (TC); $2.2 \mathrm{mS} / \mathrm{cm}^{2}$ (RE) & Low-threshold $\mathrm{Ca}^{2+}$ conductance \\
\hline$g_{h}$ & $0.016 \mathrm{mS} / \mathrm{cm}^{2}(\mathrm{TC}) ; 0 \mathrm{mS} / \mathrm{cm}^{2}(\mathrm{RE})$ & $\begin{array}{l}\text { Hyperpolarization-activated catior } \\
\text { conductance }\end{array}$ \\
\hline \multicolumn{3}{|c|}{ Cortical cells (soma) } \\
\hline$S_{\text {soma }}$ & $1.0 \times 10^{-6} \mathrm{~cm}^{2}(\mathrm{PY} ; \mathrm{IN})$ & $\begin{array}{l}\text { Area of the axosomatic } \\
\text { compartment }\end{array}$ \\
\hline$g_{K}$ & $200 \mathrm{mS} / \mathrm{cm}^{2}(\mathrm{PY} ; \mathrm{IN})$ & Maximal potassium conductance \\
\hline$g_{\mathrm{Na}}$ & $3000 \mathrm{mS} / \mathrm{cm}^{2}$ (PY); $2500 \mathrm{mS} / \mathrm{cm}^{2}$ (IN) & Maximal sodium conductance \\
\hline$g_{\mathrm{Na}(\mathrm{p})}$ & $15 \mathrm{mS} / \mathrm{cm}^{2}(\mathrm{PY}) ; 0 \mathrm{mS} / \mathrm{cm}^{2}(\mathrm{IN})$ & $\begin{array}{l}\text { Maximal persistent sodium } \\
\text { conductance }\end{array}$ \\
\hline \multicolumn{3}{|c|}{ Cortical cells (dendrite) } \\
\hline$\rho$ & $165(\mathrm{PY}) ; 50(\mathrm{IN})$ & $S_{\text {dend }}=\rho S_{\text {soma }}$ \\
\hline$G_{L}$ & $0.009 \mathrm{mS} / \mathrm{cm}^{2}(\mathrm{PY}) ; 0.009 \mathrm{mS} / \mathrm{cm}^{2}(\mathrm{IN})$ & Leakage conductance \\
\hline$E_{L}$ & $-67 \mathrm{mV}(\mathrm{PY}) ;-70 \mathrm{mV}(\mathrm{IN})$ & Leakage reversal potential \\
\hline $\mathrm{G}_{\mathrm{KL}}$ & $0.011 \mathrm{mS} / \mathrm{cm}^{2}(\mathrm{PY}) ; 0.009 \mathrm{mS} / \mathrm{cm}^{2}(\mathrm{IN})$ & Potassium leakage conductance \\
\hline$E_{K}$ & $-95 \mathrm{mV}(\mathrm{PY} ; \mathrm{IN})$ & Potassium reversal potential \\
\hline$g_{\text {Na }}$ & $0.8 \mathrm{mS} / \mathrm{cm}^{2}(\mathrm{PY} ; \mathrm{IN})$ & Maximal sodium conductance \\
\hline$g_{\mathrm{Na}(\mathrm{p})}$ & $2.5 \mathrm{mS} / \mathrm{cm}^{2}(\mathrm{PY}) ; 0 \mathrm{mS} / \mathrm{cm}^{2}(\mathrm{IN})$ & $\begin{array}{l}\text { Maximal persistent sodium } \\
\text { conductance }\end{array}$ \\
\hline$g_{\text {HVA }}$ & $0.01 \mathrm{mS} / \mathrm{cm}^{2}(\mathrm{PY} ; \mathrm{IN})$ & $\begin{array}{l}\text { Maximal high-threshold } \mathrm{Ca}^{2+} \\
\text { conductance }\end{array}$ \\
\hline$g_{\mathrm{KCa}}$ & $0.05 \mathrm{mS} / \mathrm{cm}^{2}(\mathrm{PY} ; \mathrm{IN})$ & $\begin{array}{l}\text { Slow } \mathrm{Ca}^{2+} \text {-dependent } \mathrm{K}^{+} \\
\text {conductance }\end{array}$ \\
\hline$g_{\mathrm{Km}}$ & $0.02 \mathrm{mS} / \mathrm{cm}^{2}(\mathrm{PY}) ; 0.015 \mathrm{mS} / \mathrm{cm}^{2}(\mathrm{IN})$ & $\begin{array}{l}\text { Slow voltage-dependent } \\
\text { noninactivating } \mathrm{K}^{+} \\
\text {conductance }\end{array}$ \\
\hline
\end{tabular}

${ }^{a}$ This table includes the units and description of the parameters used in the model.

\section{Materials and Methods}

\section{Model description}

Network geometry. The thalamocortical network model incorporated 40 thalamic relay (TC) and 40 reticular (RE) neurons in the thalamus, 200 pyramidal (PY) neurons and 40 inhibitory interneurons (IN) in the cortex (Bazhenov et al., 2002; Wei et al., 2016, 2018) organized with local synaptic connectivity (see Fig. 1a). The PY and IN neurons received inputs from PY neurons via AMPA and NMDA synapses, and PY neurons also received inputs from IN neurons via $\mathrm{GABA}_{\mathrm{A}}$ synapses. The radii of connections between cortical neurons were $\mathrm{R}_{\mathrm{AMPA}(\mathrm{PY}-\mathrm{PY})}=5$, $\mathrm{R}_{\mathrm{NMDA}(\mathrm{PY}-\mathrm{PY})}=5, \mathrm{R}_{\mathrm{AMPA}(\mathrm{PY}-\mathrm{IN})}=1, \mathrm{R}_{\mathrm{NMDA}(\mathrm{PY}-\mathrm{IN})}=1$, and $\mathrm{R}_{\mathrm{GABAA}(\mathrm{IN}-\mathrm{PY})}=$ 5. The TC neurons projected to RE neurons through AMPA synapses $\left(\mathrm{R}_{\mathrm{AMPA}(\mathrm{TC}-\mathrm{RE})}=8\right)$, and connections from RE to TC neurons included $\mathrm{GABA}_{\mathrm{A}}$ and $\mathrm{GABA}_{\mathrm{B}}$ synapses $\left(\mathrm{R}_{\mathrm{GABAA}(\mathrm{RE}-\mathrm{TC})}=8, \mathrm{R}_{\mathrm{GABAB}(\mathrm{RE}-\mathrm{TC})}=8\right)$. The radii of connections between $\mathrm{RE}$ and $\mathrm{RE}$ were $\mathrm{R}_{\mathrm{GABAA}(\mathrm{RE}-\mathrm{RE})}=5$. Thalamocortical connections were wider and mediated by AMPA synapses from TC neurons $\left(\mathrm{R}_{\mathrm{AMPA}(\mathrm{TC}-\mathrm{PY})}=20, \mathrm{R}_{\mathrm{AMPA}(\mathrm{TC}-\mathrm{IN})}=4\right)$; corticothalamic connections were mediated by AMPA synapses from PY neurons $\left(\mathrm{R}_{\mathrm{AMPA}(\mathrm{PY}-\mathrm{TC})}=10, \mathrm{R}_{\mathrm{AMPA}(\mathrm{PY}-\mathrm{RE})}=8\right)$. Flat connectivity profiles were used for all synaptic connections. For example, $\mathrm{R}_{\mathrm{AMPA}(\mathrm{PY}-\mathrm{PY})}=$ 5 , indicating that each PY receives AMPA synaptic input from the left five and the right five PY neurons. We previously tested different radii of connections and exponentially decaying profiles and found qualitatively similar network dynamics, assuming that synaptic connections are scaled to maintain total synaptic input per neuron. All neurons were modeled based on the Hodgkin-Huxley kinetics. The units and description of parameters are summarized in Table 1.

Neuromodulators and sleep stages. The model implemented the change of neuromodulators, such as acetylcholine (ACh), histamine (HA), and also GABA, in the intrinsic and synaptic currents to model transitions between sleep stages (Krishnan et al., 2016). Compared with the awake state, in Stage N3 sleep, the levels of ACh and HA were reduced while the 


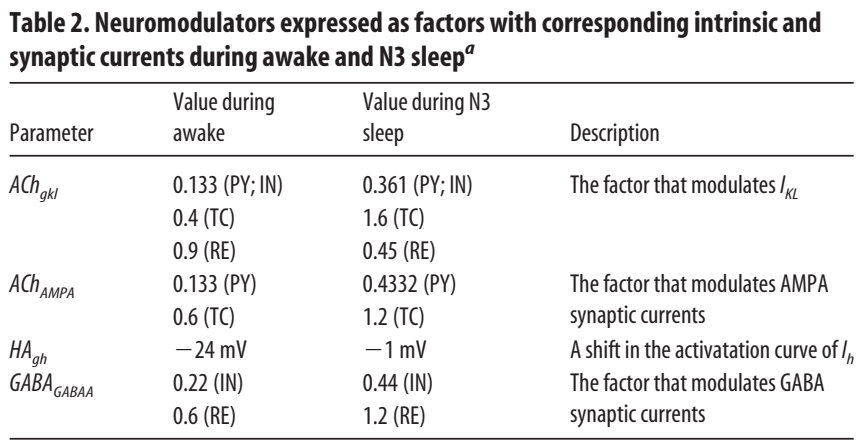

${ }^{a}$ Equations for all intrinsic and synaptic currents implementing the factors listed are provided in the corresponding Materials and Methods sections.

level of the inhibitory neurotransmitter GABA was increased. Specifically, the reduction of ACh was implemented as an increase in potassium leak conductance in TC, PY, and IN neurons, a reduction of potassium leak conductance in RE cells (McCormick, 1992), and an increase in AMPA connection strength (Kimura et al., 1999). The reduction of HA was implemented as a negative shift in the activation curve of a hyperpolarization-activated cation current $\left(I_{h}\right)$ (McCormick and Williamson, 1991; McCormick, 1992). The increase of GABA was implemented as an increase of the maximal conductance of GABAergic synapses in IN and RE neurons (Krishnan et al., 2016). Since we model effects of neuromodulators phenomenologically (Krishnan et al., 2016), we do not use the absolute values to simulate neuromodulator activity but apply a factor indicating relative change on each intrinsic or synaptic conductance compare to the awake state (Table 2). These synaptic and intrinsic changes were tuned to model transitions between awake state and SWS (N3 sleep) (Krishnan et al., 2016).

Intrinsic currents: cortex. Cortical PY and IN neurons included dendritic and axo-somatic compartments, similar to the models used in our previous papers (Timofeev et al., 2000; Bazhenov et al., 2002; Chen et al., 2012; Krishnan et al., 2016; Wei et al., 2016, 2018), representing a reduction of the multicompartmental neuron model as described previously (Mainen and Sejnowski, 1996) as follows:

$$
\begin{aligned}
C_{m} \frac{d V_{D}}{d t}=-A C h_{g k l} I_{K L}-I_{N a}-I_{N a(p)}- & I_{K m}-I_{K C a}-I_{H V A}-I_{L} \\
& -g\left(V_{D}-V_{S}\right)-I_{s y n} \\
0=-g\left(V_{S}-V_{D}\right)-I_{N a}- & I_{K}-I_{N a(p)}
\end{aligned}
$$

where $C_{m}$ is the membrane capacitance, $A C h_{g k l}$ represents the modulation on potassium leak current $I_{K L}$ based on the level of ACh during different sleep stages (Table 2), $I_{\mathrm{Na}}$ is a fast sodium current, $I_{\mathrm{Na}(\mathrm{p})}$ is a persistent sodium current, $I_{K m}$ is a slow voltage-dependent noninactivating potassium current, $I_{K C a}$ is a slow $\mathrm{Ca}^{2+}$-dependent $\mathrm{K}^{+}$current, $I_{H V A}$ is a high-threshold $\mathrm{Ca}^{2+}$ current, $I_{L}$ is the $\mathrm{Cl}^{-}$leak current, $\mathrm{g}$ is the conductance between axo-somatic and dendritic compartment, $V_{D}$ and $V_{S}$ are the membrane potentials of dendritic and axosomatic compartments, and $\mathrm{I}_{s y n}$ is the sum of synaptic currents to the neuron. This model was first proposed by Mainen and Sejnowski (1996) as a reduction of a multicompartmental pyramidal cell model, based on the assumption that the current dynamics in the axosomatic compartment are fast enough to ensure that $V_{\mathrm{S}}$ is always at equilibrium state, as defined by the second equation in Equation 1. Indeed, this reduced model has relatively high $\mathrm{Na}^{+}$and $\mathrm{K}^{+}$conductance values $\left(g_{\mathrm{Na}}=3000 \mathrm{mS} / \mathrm{cm}^{2}, g_{\mathrm{K}}=200\right.$ $\mathrm{mS} / \mathrm{cm}^{2}$ ) (Mainen and Sejnowski, 1996) in the axosomatic compartment (representing the axon hillock in the model). Therefore, the full version of the axosomatic membrane voltage equation $C d V s / d t=-g\left(V_{\mathrm{S}}-V_{\mathrm{D}}\right)$, $I_{\mathrm{S}}^{\text {int }}$ can be rewritten in a form $\varepsilon d V s / d t=F(V s)$, where $\varepsilon$ is a small parameter and $F(V s)$ represents axosomatic currents normalized to match the magnitude of the dendritic currents. Using singular perturbation analysis (Kuznetsov, 1995), we can find that the state variable Vs quickly reaches the manifold of slow motion defined by equation $F(V s)$ $=0$, which corresponds to Equation 1 in our model (see detailed discus- sion in Chen et al., 2012). The persistent sodium current $I_{N a(p)}$ was included in the axosomatic and dendritic compartment of PY cells to increase bursting propensity. IN cells had the same intrinsic currents as those in PY cells, except that $I_{\mathrm{Na}(p)}$ was not included. All the voltagedependent ionic currents $I_{j}$ have the similar form as follows:

$$
I_{j}=g_{j} m^{M} h^{N}\left(V-E_{j}\right)
$$

where $g_{j}$ is the maximal conductance, $\mathrm{m}$ and $\mathrm{h}$ are gating variables, $\mathrm{V}$ is the voltage of the corresponding compartment, and $E_{j}$ is the reversal potential. The dynamic of gating variables are described as follows:

$$
\begin{gathered}
\frac{d x}{d t}=-\frac{x-x_{\infty}}{\tau_{x}} \\
\tau_{x}=\left(1 /\left(\alpha_{x}+\beta_{x}\right)\right) / Q_{T} \\
x_{\infty}=\alpha_{x} /\left(\alpha_{x}+\beta_{x}\right)
\end{gathered}
$$

where $x=\mathrm{m}$ or h. $\mathrm{Q}_{\mathrm{T}}$ is a temperature-related term, $\mathrm{Q}_{\mathrm{T}}=\mathrm{Q}^{((\mathrm{T}-23) / 10)}=$ 2.9529 , with $\mathrm{Q}=2.3, \mathrm{~T}=36^{\circ} \mathrm{C}$. The detailed description of individual currents was provided in our previous study (Wei et al., 2016).

Intrinsic currents: thalamus. The thalamic TC and RE cells were modeled as a single compartment that included voltage- and calciumdependent currents described by the Hodgkin-Huxley kinetic (Bazhenov et al., 2002) as follows:

$$
C_{m} \frac{d V}{d t}=-A C h_{g k l} I_{K L}-I_{N a}-I_{K}-I_{T}-I_{h}-I_{L}-I_{s y n}
$$

where $A C h_{g k l}$ in TC cells and RE cells is summarized in Table 2. $I_{K L}$ is a potassium leak current, $I_{\mathrm{Na}}$ is a fast sodium current, $I_{K}$ is a fast potassium current, $I_{T}$ is a low-threshold $\mathrm{Ca}^{2+}$ current, $I_{h}$ is a hyperpolarizationactivated cation current, $I_{L}$ is a Cl${ }^{-}$leak current, and $I_{s y n}$ is the sum of the synaptic currents to the neuron. The hyperpolarization-activated cation current $I_{h}$ was only included in TC neurons, not in RE neurons. The detailed description of individual currents was provided in our previous study (Wei et al., 2016). The effect of HA on $I_{h}$ was implemented as a shift of $H A_{g h}$ in the activation curve (Krishnan et al., 2016) as follows:

$$
m_{\infty}=1 /\left(1+\exp \left(\left(V+75+H A_{g h}\right) / 5.5\right)\right)
$$

where $H A_{g h}$ is summarized in Table 2.

Synaptic currents. The equations for $\mathrm{GABA}_{\mathrm{A}}$, AMPA, and NMDA synaptic currents were described by first-order activation schemes, and the $\mathrm{GABA}_{\mathrm{B}}$ synaptic currents had a more complex scheme of activation that involved the activation of $\mathrm{K}^{+}$channels by $\mathrm{G}$ proteins (Destexhe et al., 1996). The equations for all synaptic currents used in this model were given in our previous studies (Bazhenov et al., 2002; Wei et al., 2016). In this paper, we added the level of ACh and GABA to modulate AMPA, and $\mathrm{GABA}_{\mathrm{A}}$ synaptic currents as described by the following:

$$
\begin{gathered}
I_{s y n}^{A M P A}=A C h_{A M P A} g_{s y n}[O]\left(V-E_{s y n}\right) \\
I_{s y n}^{G A B A}=G A B A_{G A B A A} g_{s y n}[O]\left(V-E_{s y n}\right)
\end{gathered}
$$

where $\mathrm{g}_{\text {syn }}$ is the maximal conductance, $[\mathrm{O}]$ is the fraction of open channels, and $\mathrm{E}_{\mathrm{syn}}$ is the reversal potential $\left(\mathrm{E}_{\mathrm{AMPA}}=0 \mathrm{mV}, \mathrm{E}_{\mathrm{NMDA}}=0 \mathrm{mV}\right.$, and $\left.\mathrm{E}_{\mathrm{GABAA}}=-70 \mathrm{mV}\right) . \mathrm{ACh}_{\mathrm{AMPA}}$ is the variable that modulates AMPA synaptic currents for cortical PY-PY, TC-PY, and TC-IN connections by the level of ACh. $\mathrm{ACh}_{\mathrm{AMPA}}$ from PY and TC cells is summarized in Table 2. $\mathrm{GABA}_{\mathrm{GABAA}}$ is the variable that modulates GABA synaptic currents for cortical IN-PY, RE-RE, and RE-TC connections. GABA ${ }_{\text {GABAA }}$ from IN and RE cells is summarized in Table 2. The maximal conductance for each specific synapse type was as follows: $\mathrm{g}_{\mathrm{GABAA}(\mathrm{RE}-\mathrm{TC})}=0.06 \mu \mathrm{S}$, $\mathrm{g}_{\mathrm{GABAB}(\mathrm{RE}-\mathrm{TC})}=0.0025 \mu \mathrm{S}, \mathrm{g}_{\mathrm{GABAA}(\mathrm{RE}-\mathrm{RE})}=0.1 \mu \mathrm{S}, \mathrm{g}_{\mathrm{AMPA}(\mathrm{TC}-\mathrm{RE})}=0.06$ $\mu \mathrm{S}, \mathrm{g}_{\mathrm{AMPA}(\mathrm{TC}-\mathrm{PY})}=0.14 \mu \mathrm{S}, \mathrm{g}_{\mathrm{AMPA}(\mathrm{TC}-\mathrm{IN})}=0.12 \mu \mathrm{S}, \mathrm{g}_{\mathrm{AMPA}(\mathrm{PY}-\mathrm{PY})}=$ $0.24 \mu \mathrm{S}, \mathrm{g}_{\mathrm{NMDA}(\mathrm{PY}-\mathrm{PY})}=0.01 \mu \mathrm{S}, \mathrm{g}_{\mathrm{AMPA}(\mathrm{PY}-\mathrm{IN})}=0.12 \mu \mathrm{S}, \mathrm{g}_{\mathrm{NMDA}(\mathrm{PY}-\mathrm{IN})}$

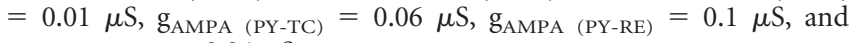
$\mathrm{g}_{\mathrm{GABAA}(\mathrm{IN}-\mathrm{PY})}=0.24 \mu \mathrm{S}$.

In addition, spontaneous miniature EPSPs and IPSPs were implemented for PY-PY, PY-IN, and IN-PY connections. The arrival times of 
spontaneous miniature EPSPs and IPSPs were modeled by Poisson processes (Stevens, 1993), with time-dependent mean rate $\mu=(2 /(1+$ $\left.\left.\exp \left(-\left(t-t_{0}\right) / F\right)\right)-1\right) / 250$ (Bazhenov et al., 2002), where $t_{0}$ is a time instant of the last presynaptic spike (Timofeev et al., 2000). The mEPSP frequency $(\mathrm{F})$ and amplitude $(\mathrm{A})$ were $\mathrm{F}_{\mathrm{PY}-\mathrm{PY}}=30, \mathrm{~F}_{\mathrm{PY}-\mathrm{IN}}=30, \mathrm{~F}_{\mathrm{IN}-\mathrm{PY}}=$ $30, \mathrm{~A}_{\text {PY-PY }}=0.2 \mathrm{mV}, \mathrm{A}_{\mathrm{PY}-\mathrm{IN}}=0.2 \mathrm{mV}$, and $\mathrm{A}_{\mathrm{IN} \text {-PY }}=0.2 \mathrm{mV}$.

$S T D P$. Facilitation or depression of the synaptic strength is believed to underlie learning in the brain. Here we used STDP model of synaptic plasticity to adjust the synaptic connections between cortical pyramidal neurons based on the relative timing of the presynaptic and postsynaptic spikes. The change of excitatory synaptic connections $\left(\mathrm{g}_{\mathrm{AMPA}}\right)$ and the amplitude of mEPSC $\left(\mathrm{A}_{\mathrm{mEPSC}}\right)$ were described as in our previous paper (Wei et al., 2016) as follows:

$$
\begin{gathered}
g_{A M P A} \leftarrow g_{A M P A}+g_{\text {max }} F(\Delta t) \\
A_{m E P S C} \leftarrow A_{m E P S C}+f A_{P_{Y_{-} P Y} F} F(\Delta t)
\end{gathered}
$$

where $g_{\max }$ is the maximal synaptic conductance of $\mathrm{g}_{\text {AMPA }} . f=0.01$ is a scaling factor. $F$ is the STDP function that shows the change of synaptic connections as a function of the relative timing $(\Delta t)$ of presynaptic and postsynaptic spikes (Song et al., 2000), as follows:

$$
F(\Delta t)=\left\{\begin{array}{l}
A_{+} e^{-\frac{|\Delta t|}{t_{+}}}, \text {if } \Delta t>0 \\
-A_{-} e^{-\frac{|\Delta t|}{t_{-}}}, \text {if } \Delta t<0
\end{array}\right.
$$

where parameters $\mathrm{A}_{+}$and $\mathrm{A}_{-}$determine the maximum amounts of synaptic modification. Here, we set $\mathrm{A}_{+}=\mathrm{A}_{-}=0.002$, and $\tau_{+}=\tau_{-}=20 \mathrm{~ms}$. We reduced the STDP amplitude $\mathrm{A}_{+}$and $\mathrm{A}_{-}$to 0.001 during SWS to account for reduction of ACh (Sugisaki et al., 2015). We assumed that the synaptic efficacy should stay within $[0,200 \%]$ range of the initial synaptic weights to prevent STDP from runaway synaptic dynamics. We would like to note that in vivo the rate of synaptic potentiation is slower than that in the model and typically saturates $\sim 150 \%$ of cortical neurons over a full night (Chauvette et al., 2012). Because of that, although our simulation times (in absolute unites) are much shorter than a full night, the change of the synaptic weights in the trained region was sufficient to observe the performance improvement after sleep.

Training and test. For most of the simulations, the training pattern included 5 groups of neurons that were activated in sequential order, with $5 \mathrm{~ms}$ delays between subsequent groups of activation. Each group was a set of 5 adjacent neurons drawn from a contiguous 25 cell subregion of the full 200 cell network. For example, if the sequence started at neuron \#50, these 5 groups were as follows: A (\#50-54), B (\#55-59), C $(\# 60-64), \mathrm{D}(\# 65-69)$, and $\mathrm{E}(\# 70-74)$. Each group was stimulated by a step current that led to a suprathreshold response with a duration of 10 $\mathrm{ms}$ and a delay of $5 \mathrm{~ms}$ between groups. Thus, during training, the neuronal activity in these groups reflected the order of the trained sequence (e.g., ABCDE). During test sessions, the model was only presented with the first input at Group A to recall the trained sequence ABCDE within a $350 \mathrm{~ms}$ response window. During both training and test sessions, each trial was repeated every $1 \mathrm{~s}$.

Stimulation protocols. To model sensory stimulation, we modeled independently a second (sensory) cortical network with 200 PY cells that were connected one-to-one to the PY cells of the primary network. We mimicked two different types of the auditory stimulation. For the learning-specific cue, we hypothesized that the sensory cue only affects a small population of neurons (local cue). The stimulation was applied at the local site Q (\#50-54) of the secondary cortical network, which projected to the specific location A (\#50-54) of the primary network. During the training session, we stimulated sequentially $\mathrm{Q} \rightarrow \mathrm{A} \rightarrow \mathrm{B} \rightarrow \mathrm{C} \rightarrow \mathrm{D} \rightarrow \mathrm{E}$. The pink noise auditory stimulation can potentially activate a broad group of neurons (broad cue). In this protocol, the stimulation was applied during sleep to the broad population of neurons Q (\#30-169) of the secondary cortical network, which activated a broad region (\#30169) of the primary network.

During sleep, in the open-loop stimulation protocol, the cue lasted for $50 \mathrm{~ms}$ and was repeated at a frequency of $0.75 \mathrm{~Hz}$, which was close to the internal frequency of SO in the model. In the closed-loop stimulation protocol, we first detected the onset of the Down state or Up state in ongoing SO, then presented the cue with X ms delay after Down or Up state onset as defined below. We set X to be $0,100,200,300,400$, or 500 ms. The Up or Down states were detected based on the analysis of the local field potential (LFP). The LFP was approximated by calculating mean membrane potentials of all the cortical excitatory neurons, and it had a bimodal distribution during N3 sleep, where one peak corresponded to the Up state and another peak to the Down state (see Fig. $1 f$ ). The trough of the distribution was selected as a threshold to separate Up and Down state. The onset of Up or Down state was then defined as the moment when LFP value crossed the threshold. In the closed-loop Best Phase protocol, the stimulation was delivered at the optimal phase predicted by the model (that gives the highest performance after sleep), which corresponds to $\sim 500 \mathrm{~ms}$ after detecting the onset of a Down state, at each cycle of SO. In the closed-loop 2-Click protocol, the stimulation was delivered at the optimal phase at two sequential cycles of SO with a pause of $2.5 \mathrm{~s}$ after the second stimulation to match experimental protocol (Ngo et al., 2015).

Computational methods. All model simulations were performed using a fourth-order Runge-Kutta integration method with a time step of 0.02 $\mathrm{ms}$. Source $\mathrm{C}++$ was compiled on a Linux server using the gcc compiler. Part of the simulation was run on the Neuroscience Gateway (Sivagnanam et al., 2013). All data processing was done with custom-written programs in MATLAB (The MathWorks). The model simulation code will become available from https://www.bazhlab.ucsd.edu/downloads/ after publication.

\section{Data analysis}

Sequence learning analysis. To model sequence learning, the model was presented with multiple trials of sequential input to the groups of selected cortical neurons. The performance of sequence recall was measured by the percentage of success of sequence recall during test sessions when only the first group of a sequence was stimulated. First, we detected the network sequence using the following steps: (1) We detected all spikes for five groups of neurons (each group contains five neurons) within a 350 $\mathrm{ms}$ response time window (starting from the time when test stimulus was applied). (2) We smoothed the firing rate of each group by convoluting the average instantaneous firing rate of five neurons with a Gaussian kernel (50 ms window size). (3) The firing sequence of the groups was determined by ordering the peaks of their smoothed firing rates during a $350 \mathrm{~ms}$ window. Next, we applied a String Match (SM) method to measure the similarity between each detected sequence and an ideal sequence (e.g., $S=\mathrm{ABCDE}$ ). The $\mathrm{SM}$ was calculated as $S M=2 * \mathrm{~N}$ $-\sum_{i=1}^{N}\left|L\left(S_{1}, S_{2}[i]\right)-i\right|$, where $S_{1}$ is the test sequence generated by the network, $S_{2}$ is the subset of ideal sequence that only contains the same elements as $S_{1}, N$ is the sequence length of $S_{1}$, and $L\left(S_{1}, S_{2}[i]\right)$ represents the location of element $S_{2}[i]$ in a sequence $S_{1}$. SM was then normalized by dividing by $M$, where $M$ is 2 times the length of $S$. For example, if the ideal sequence $S$ was $\mathrm{ABCDE}$ and $S_{1}$ was $\mathrm{ACDB}$, then $S_{2}=\mathrm{ABCD}, N=4$. The location of element $A$ in $S_{1}$ is $L\left(S_{1}, A\right)=1 ; B$ in $S_{1}$ is $L\left(S_{1}, B\right)=4$; $C$ in $S_{1}$ is $L\left(S_{1}, C\right)=2$; and $D$ in $S_{1}$ is $L\left(S_{1}, D\right)=3$. Therefore, $\mathrm{SM}=2 \times 4-(\mid 1-$ $1|+| 4-2|+| 2-3|+| 3-4 \mid)=4$. After SM was normalized by $\mathrm{M}=$ 10 , it became 0.4 , indicating the recalled sequence has $40 \%$ similarity to the ideal sequence. If the ideal sequence $S$ was $\mathrm{ABCDE}$ and $S_{1}$ was $\mathrm{ABCDE}$, then $S_{2}=\mathrm{ABCDE}, N=5$, and $\mathrm{SM}=2 \times 5-0=10$, or 1.0 after normalization by 10 . The performance was calculated as the percentage of recalled sequences with $S M \geq T$ during the test session. In this paper, we selected a threshold of $\mathrm{Th}=0.8$, indicating that a recalled sequence with at least $80 \%$ similarity to the ideal sequence was counted as a successful recall. Baseline performance (before training) of the network was $\sim 15 \%$ for $\mathrm{Th}=0.8$ due to the random spiking. If higher threshold $\mathrm{Th}=$ 1.0 was selected, the baseline performance became almost 0 .

Analysis of synaptic weights. Synaptic weights between neurons in a direction of sequence activation were enhanced due to the sequence replay. The mean of the changes of synaptic weights associated with a given sequence was used to characterize memory strength.

Phase locking index (PLI). The PLI was applied to quantify the phase synchronization between the LFP signals. It was calculated based on the following equation: 
a

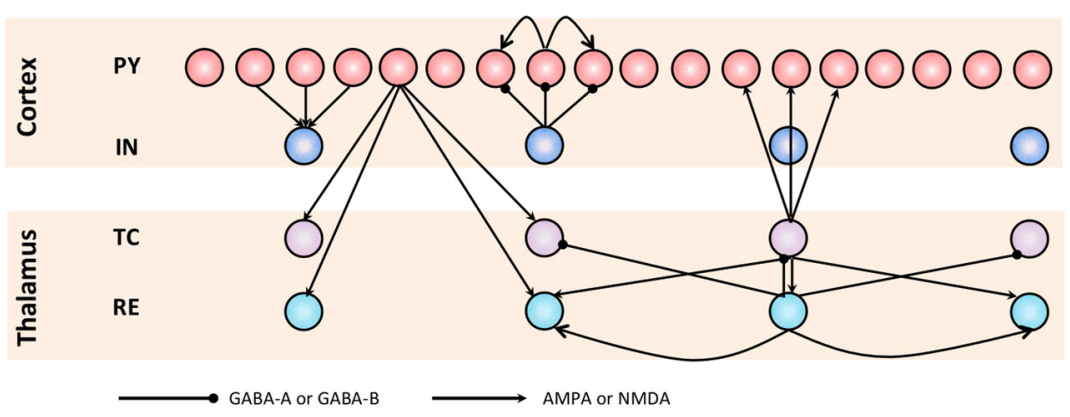

b

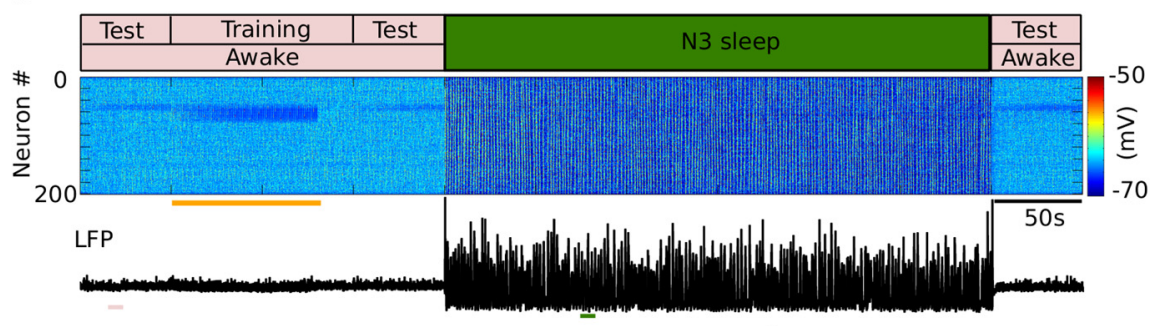

C
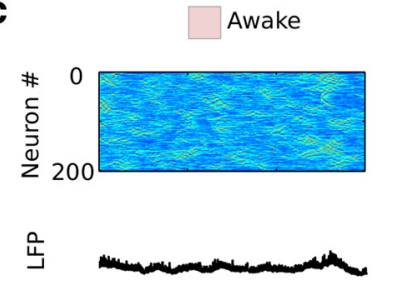

Slow oscillations
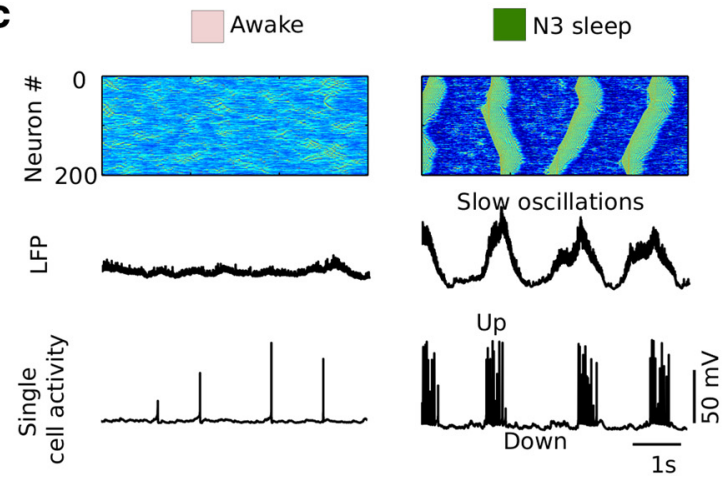

d
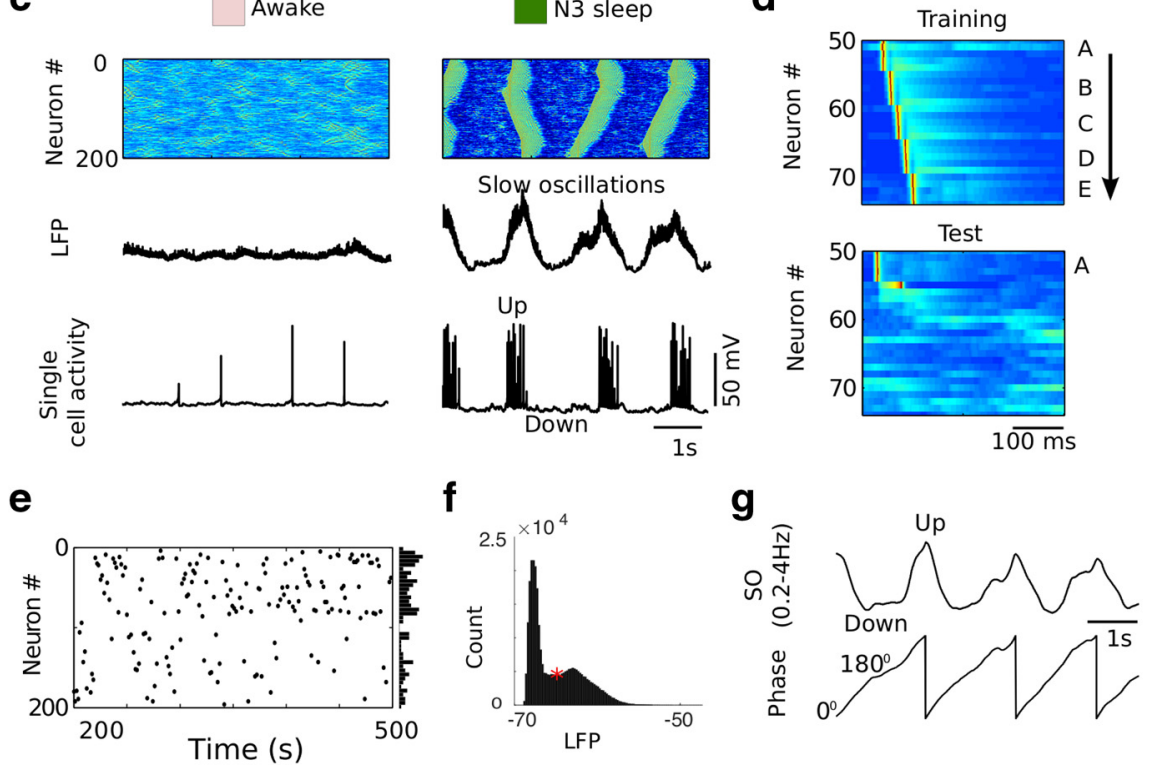

Figure 1. Network dynamics and sequence learning paradigm in the model. $\boldsymbol{a}$, The network geometry and connectivity structure. $\boldsymbol{b}$, The cortical network activity during transition from the awake state (pink block, top), to N3 sleep (SWS) (dark green block) and back to awake. Raster plot (middle) represents membrane voltages of the cortical pyramidal cells. LFP (bottom) calculated from the cortical population. The sequence was learned during the training period ( $80 \mathrm{~s}$, orange bar). Performance was tested in three test sessions: before training, after training before sleep, and after sleep. $\boldsymbol{c}$, The expanded view of characteristic spatiotemporal patterns (top), LFP (middle), and single-cell activity (bottom) of neuron \#50 during awake (left), and N3 sleep (right) from where pink and dark green bars are shown under LFP traces in $\boldsymbol{b}$. The SOs $(<1 \mathrm{~Hz})$ during N3 sleep consist of a typical Up and Down state transition. $\boldsymbol{d}$, The characteristic examples of a training session and a test session. Training included stimulating sequentially: Groups A-E (each group was stimulated for $10 \mathrm{~ms}$, and the delay between groups was $5 \mathrm{~ms}$ ). The test included stimulating only Group A to recall the trained sequence within a $350 \mathrm{~ms}$ response window. The sequence started at neuron \#50. Each group included five PY neurons. $\boldsymbol{e}$, Black dots represent Up state initiation sites over the entire sleep period. Right, Vertical panel represents probability of Up state initiation across neurons. $\boldsymbol{f}$, The LFP distribution during N3 sleep. Red star indicates the trough of the distribution. $\boldsymbol{g}$, The relationship between $\mathrm{SO}$ waveform and its calculated phase.

$$
P L I=\frac{1}{N}\left|\sum e^{\varnothing 1}-e^{\varnothing 2}\right|
$$

where $\varnothing 1$ and $\varnothing 2$ were the Hilbert transforms of the two signals. In this paper, global LFP was calculated as the population activity from all 200 cortical PY cells, whereas the focal LFP was calculated as the population activity from adjacent 25 cortical PY cells selected at the different network locations. To quantify how external stimulation affects the spatiotemporal pattern of SO, we calculated the averaged PLI between each two combinations of the focal LFPs during individual Up states. The higher PLI indicates the higher spatial synchronization of the network activity due to the stimulation.

\section{Statistical analysis}

When data were normally distributed based on the Anderson-Darling test, numerical values are given as mean \pm SEM (the standard error of the mean). Otherwise, we used median \pm interquartile range to report the data. For each experiment, 20 simulations with different random seeds were performed. If data had a normal distribution, the parametric test was used; otherwise, the equivalent nonparametric test was applied. If only two groups of data were compared, the two-sample $t$ test (parametric) or the Mann-Whitney $U$ test (nonparametric) was used. When data were paired, nonparametric Wilcoxon signed rank test was used. When more than two groups of data were compared, one-way ANOVA (parametric) or Kruskal-Wallis ANOVA test (nonparametric) with Bonferroni's post hoc test was applied. To compare the means of two or more columns and two or more rows of the observations, two-way ANOVA was used.

\section{Results}

The model presented in this new study is built upon the models we used in our earlier work (Krishnan et al., 2016; Wei et al., 2016, 2018). In short, the base model (Fig. 1a) represents minimal thalamocortical architecture implementing one cortical layer (consisting of PY and IN neurons) and one thalamic layer (consisting of TC and RE neurons), with all the neurons simulated using one- or two-compartmental HodgkinHuxley models. This network structure is a simplification over the three cortical layer model used previously (Wei et al., 2016), but it implements effects of neuromodulators (Krishnan et al., 2016; Wei et al., 2018) to simulate transitions between sleep and wake states (Fig. 1b). Furthermore, the new model includes a cortical sensory network for simulating effects of auditory stimulation. Synapses are based on AMPA, NMDA, $\mathrm{GABA}_{\mathrm{A}}$, and $\mathrm{GABA}_{\mathrm{B}}$ receptors with connectivity patterns identical to those used in our previous work (Krishnan et al., 2016; Wei et al., 2016, 2018).

\section{Periodic stimulation during sleep enhances memory performance}

The characteristic network activity during the awake state (Fig. $1 c$, left) reveals random spiking and fluctuations in the LFPs, whereas during N3 sleep the network displays slow $(<1 \mathrm{~Hz})$ oscillations (Fig. 1c, right) characterized by repetitive transitions between Up and Down states in all cortical neurons (Blake and Gerard, 1937; Steriade et al., 1993, 2001). Similar to Wei et al. (2018), the awake state includes one training and three test sessions: before training, after training before sleep, and after sleep (Fig. 1b). During the training session, the model was presented 
with a sequence of stimuli applied to selected groups of cortical neurons (Fig. $1 d$, top). Each group contained five neurons, A (\#50-54), B (\#55-59), C (\#6064), D (\#65-69), and $\mathrm{E}(\# 70-74)$, which were stimulated by $10 \mathrm{~ms}$ direct current steps that led to a suprathreshold response. A $5 \mathrm{~ms}$ delay was included between stimulations of subsequent groups. This stimulation was designed to simulate input from the other cortical areas associated with specific training protocol. Spike-timing-dependent synaptic plasticity (STDP) during training changed synaptic connectivity. In result, the model learned a sequence (e.g., ABCDE). During test sessions, the model was only presented with the input at the first Group A to test for pattern completion of the trained sequence ABCDE (Fig. 1d, bottom). During training or test sessions, each trial was repeated every $1 \mathrm{~s}$. Initiation of Up states was random in the naive network and was biased to the trained network site after sequence learning (Fig. $1 e$ ). The LFP was approximated by calculating mean membrane potentials of all the cortical excitatory neurons, and it had a bimodal distribution during N3 sleep, where one peak corresponded to the Up state and another peak corresponded to the Down state (Fig. 1f). The instantaneous phase of the ongoing SOs was derived from a Hilbert transformation of LFP filtered within the SO frequency range of $0.2-4 \mathrm{~Hz}$ : the peak of the Up state is defined as $0^{\circ}$ after Hilbert transformation, and the peak of the Down state as $180^{\circ}$ (Fig. $1 g$ ).

To model sensory cues, we modeled independently a second ("sensory") cortical network making one-to-one connections to the primary network. Thus, during the training session, we stimulated sequentially $\mathrm{Q} \rightarrow \mathrm{A} \rightarrow \mathrm{B} \rightarrow \mathrm{C} \rightarrow \mathrm{D} \rightarrow \mathrm{E}$, where group of neurons $(\mathrm{Q})$ belonged to the "sensory" network. During sleep, in an open-loop stimulation protocol, the cue lasted for $50 \mathrm{~ms}$ and was repeated at a predefined frequency of $0.75 \mathrm{~Hz}$ that was close to the internal frequency of SOs in the intact model (Fig. 2a). The cue (Fig. 2b, top, red arrow) could trigger ABCDE sequence replay (Fig. $2 d$ ); and if it occurred during the later part of the Down state, it could also initiate an Up state of the SO (Fig. $2 b$, bottom).

The phase of the SO at the time of a cue presentation (Fig. $2 b$, middle, red dots) was extracted to construct the circular histogram of the stimulation phase. The distribution had a wellformed peak (Fig. 2c), indicating that the spontaneous SO was entrained or phase-locked to the periodic cue stimulus. Analysis of the sequence recall performance among all three test sessions (Fig. 2e, gray bars) revealed the following (one-way ANOVA; $\left.F_{(2,57)}=68.49, p=6.95 \times 10^{-16}\right)$. First, repetitive training for $80 \mathrm{~s}$ during awake improved performance compared with the a The learning-related cue was presented during sleep

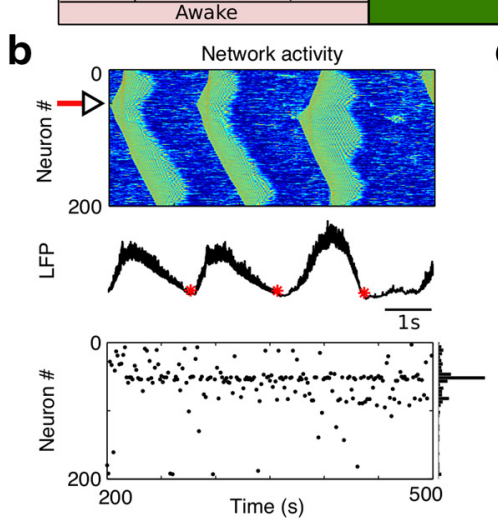

Circular histogram of phase

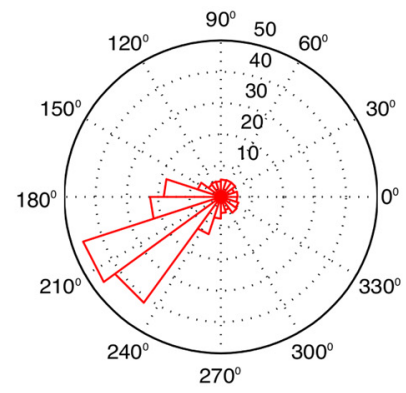

C Performance during test sessions

d Examples of sequence replay
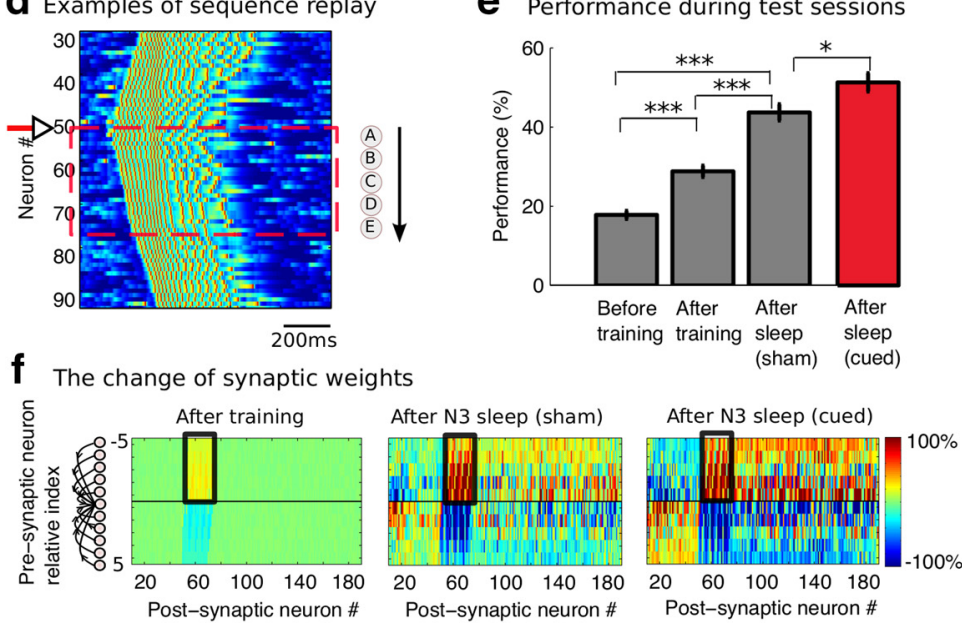

Figure 2. Periodic cue presentation during sleep. $\boldsymbol{a}$, The cue was associated with the sequence during the training session in awake. During sleep, this learning-related cue was presented for $50 \mathrm{~ms}$ every $1.333 \mathrm{~s}(\sim 0.75 \mathrm{~Hz})$ during the entire sleep period. Red lines indicate when the cue was presented. $\boldsymbol{b}$, Top, Characteristic example of network activity during SOs. Red arrow indicates cue delivered to Group A. Middle, Characteristic example of LFP. Red dots represent the times of cue presentation. Bottom, Black oss the network. $\boldsymbol{c}$, Circular histogram of SO phases at which the cue was applied. $\boldsymbol{d}$, Characteristic example of slow wave sequence $(A B C D E)$ replay when the learning-related cue was presented. $\boldsymbol{e}$, The bar plot of performance that was defined Error bars indicate SEM. ${ }^{*} p<0.05{ }^{* * *} p<0.001 . f$ The change of synaptic weights relative to the initial values after (left), after N3 sleep without cue presentation (middle), and after N3 sleep with periodic cue presentation (right). $x$ axis postsynaptic neurons at the $x$ axis. The synaptic weights between neurons in direction of sequence activation (black box) were enhanced due to training during awake (left) and sequence replay during sleep (right). Warm colors (e.g., red) represent increased synaptic weight relative to cold colors (e.g., blue).

baseline performance before training $(28.8 \pm 1.454 \%$ vs $17.8 \pm$ $1.025 \%, p=2.0677 \times 10^{-5}$, one-way ANOVA Bonferroni corrections). Second, performance after sleep was enhanced significantly compared with that before sleep without cue stimulation $\left(43.7 \pm 2.059 \%\right.$ vs $28.8 \pm 1.454 \%, p=2.926 \times 10^{-8}$, one-way ANOVA Bonferroni corrections). Finally, when the cue was presented during sleep, performance after sleep (Fig. $2 e$, red bar) was significantly higher compared with the uncued experiments $\left(51.3 \pm 2.314 \%\right.$ vs $43.7 \pm 2.059 \%, t_{(38)}=2.454, p=0.0188$, two-sample $t$ test).

To identify the change of the network connectivity underlying performance increase, we next analyzed the dynamics of synaptic weights between the cortical neurons. During the training phase, the ordered firing of neurons led to the potentiation of synapses in the direction of the trained sequence (Fig. $2 f$, left, black box), 

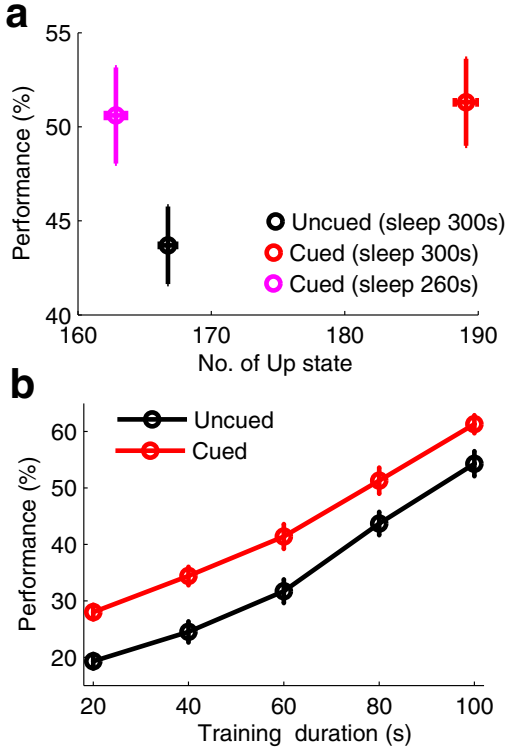

Figure 3. The effect of periodic cue presentation during sleep. $\boldsymbol{a}$, The number of Up states during sleep versus performance after sleep. Duration of the entire sleep period was either 300 or 260 s. $\boldsymbol{b}$, Performance after sleep for the different training durations (memory strengths) with (red) or without (black) cue presented during sleep. Error bars indicate SEM.

whereas the synapses corresponding to the opposite direction were depressed (Fig. $2 f$, left). These changes in the connectivity matrix among the trained neurons (Fig. $2 f$, left, black box) were augmented after the subsequent N3 sleep in both uncued (Fig. $2 f$, middle, black box) and cued experiments (Fig. $2 f$, right, black box), similar to our previous analysis of the replay without cues (Wei et al., 2018). However, the changes in the experiments with sensory cue stimulation (Fig. $2 f$, right) were higher, which explains the higher performance of memory recall after the cued sleep compared with the uncued sleep.

The number of Up states of SO was significantly increased during cued sleep compared with uncued experiments (189.1 \pm 0.85 vs $166.75 \pm 0.615, t_{(38)}=-21.32, p=9.64 \times 10^{-23}$, twosample $t$ test; Fig. $3 a$ red vs black). This is consistent with the data of the recent experiments where the greater amount of SOs was found to be elicited with the targeted sound compared with the experiments with a control new sound (Oyarzun et al., 2017). To examine whether the enhanced performance in our simulations was merely due to the higher Up state count, we reduced the sleep duration from 300 to 260 s for the cued sleep to obtain a comparable Up state count to the uncued sleep. We observed that the performance after the $260 \mathrm{~s}$ of cued sleep was still significantly higher than after the $300 \mathrm{~s}$ of uncued sleep ( $50.6 \pm 2.559 \%$ vs $43.7 \pm 2.059 \%, t_{(38)}=-2.1, p=0.0423$, two-sample $t$ test; Fig. $3 a$, pink vs black). This suggests that the performance improvement due to cue presentation can be summed up in two factors: (1) facilitation of the trained sequence replay within each Up state and (2) increase in the number of Up state events.

To characterize how relative strength of a memory trace before sleep influences the sequence recall and memory performance after the sleep, we varied duration of initial training (Fig. $3 b)$. As the training duration (memory strength before sleep) increased, the performance after sleep increased as well (Fig. $3 b$; comparison among different memory strengths, $F_{(4,190)}=$ 106.33, $p=2.2 \times 10^{-47}$, two-way ANOVA). In all cases, performance of the sequence recall after sleep was significantly higher when the cue was presented during sleep (Fig. 3b, compare black and red; $F_{(1,190)}=51.60, p=1.5 \times 10^{-11}$, two-way ANOVA). Thus, the cues presented during sleep can benefit memory consolidation at different levels of the presleep memory strength.

\section{Closed-loop stimulation protocol revealed phase-dependent responses during SWS}

Experimental studies suggest that stimuli presented at certain phases of the sleep SO have a stronger effect on memory consolidation compared with the other stimulation phases (Batterink et al., 2016). To investigate the relationship between the phase angle of the $\mathrm{SO}$ at the cue presentation and the change in performance, we used a closed-loop stimulation protocol to vary the timing of stimulation in respect to the phase of SO. In the closed-loop conditions, we first detected the onset of the Down state or Up state in an ongoing SO, then presented the cue with $\mathrm{X}$ ms delay after Down or Up state onset (see Materials and Methods). The onset of Up or Down state was defined as the moment when LFP crossed the threshold that was defined as the trough that separates two peaks in the histogram of the LFP distribution (see Materials and Methods). We set X to be $0,100,200,300,400$, or $500 \mathrm{~ms}$. In the example shown in Figure $4 a$, the cue was presented with 500 ms delay after the network transition from Up to Down state (Fig. $4 a$, left middle, red dot). After the experiment was completed, we plotted the circular histogram of stimulation phase for all stimulation events (Fig. $4 a$, right). In this particular example, the peak of the circular histogram was $\sim 210^{\circ}$ (Fig. $4 a$, right), which corresponds to the very end of the Down state of the SO. We propose that, since this was a time moment when the network was "almost ready" to start a new Up state on its own, stimulation reliably triggered a new Up state; and because of the stimulus location, these Up states were largely initiated near the beginning of the trained sequence (Group A), thus promoting sequence replay (Fig. $4 a$, left bottom, see peak of histogram). In another characteristic example in the Figure $4 b$, the cue was presented $200 \mathrm{~ms}$ after the onset of Up state (Fig. $4 b$, left middle, red star). At that phase of stimulation, the entire network was already in the Up state (phase $\sim 330^{\circ}$ ) (Fig. $4 b$, right), therefore, stimulation had minimal impact on the network spatiotemporal dynamics (Fig. $4 b$, left bottom)

Figure 4 summarizes results for all stimulation phases. When the cue was presented during the Down state $\left(90^{\circ}-270^{\circ}\right)$ of $\mathrm{SO}$, the performance (Fig. $4 c$, middle top), the synaptic weight change associated with the trained sequence replay (Fig. $4 c$, middle bottom), and the probability of Up state initiation at Group A of neurons (Fig. $4 c$, bottom) were all higher than those when the cue was presented during the Up state $\left(270^{\circ}-360^{\circ}\right.$ and $\left.0^{\circ}-90^{\circ}\right)$ and also higher than those in the uncued model (Fig. $4 c$, gray lines). The optimal phase was $\sim 210^{\circ}$, which was right before transition from Down to Up state, and it maximized synaptic changes and performance improvement (Fig. $4 c$ ). It is worth noting that, because of the relatively small size of the model, open-loop periodic stimulation at the frequency close to the natural frequency of slow waves entrained the entire network oscillation by selecting the same optimal phase of stimulation as we report here using closed-loop protocol (compare Fig. $2 c$ and Fig. $4 a$ ).

Analysis of the Up state initiation sites (see examples in Fig. $4 a, b$, bottom right, vertical histograms) revealed that, in the model with optimal stimulation phase (Fig. 4a), stimulation leads to the majority of Up states being initiated at the location corresponding to the beginning of the trained sequence, which promotes sequence replay. In contrast, for suboptimal stimulation time (Fig. 4b), distribution of the Up state initiation sites remains random. Our modeling findings are in agreement with 
a The local cue applied $500 \mathrm{~ms}$ after the onset of Down state

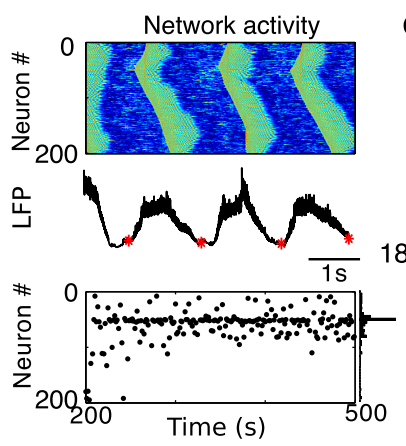

Circular histogram of phase

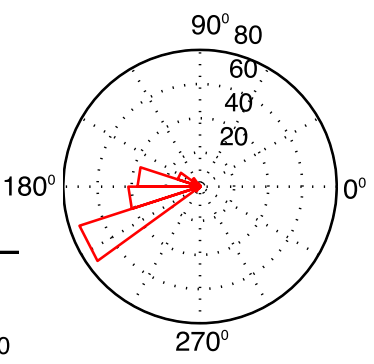

b The local cue applied $200 \mathrm{~ms}$ after the onset of Up state
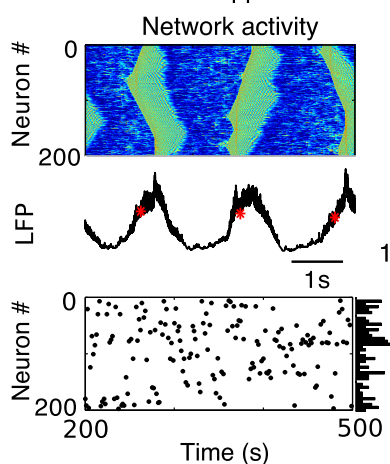

Circular histogram of phase

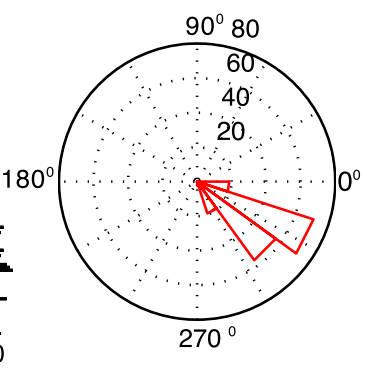

C
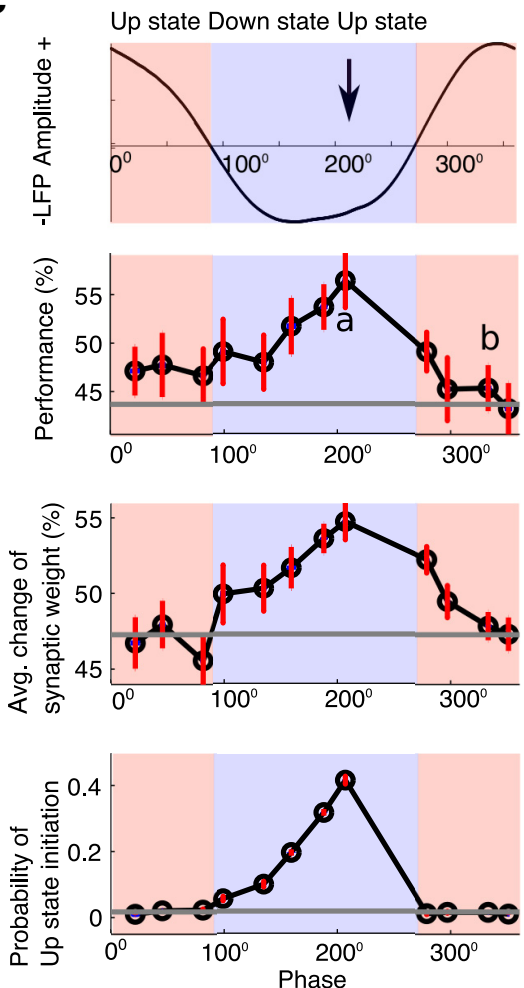

Figure 4. A closed-loop stimulation protocol during SO. $\boldsymbol{a}, \boldsymbol{b}$, Examples of cue presentation for two different phases of SO. Cue presentations in $\boldsymbol{a}$ and $\boldsymbol{b}$ correspond to $500 \mathrm{~ms}$ after the Up to Down state transition and $200 \mathrm{~ms}$ after the Down to Up state transition, respectively. $\boldsymbol{a}, \boldsymbol{b}$, Top left, Characteristic example of network activity during SO. Middle left, Characteristic example of LFP. Red stars represent the times of stimulation. Bottom left, Black dots represent Up state initiation sites during the entire sleep period. Right, Vertical panel represents probability of Up state initiation across neurons. Right, Circular histogram of phases at which the cue was applied. c, Top, The correspondence between phase angle and states of SO (Up and Down states). Vertical arrow indicates the optimal phase for cue presentation. Middle Top, The recall performance after sleep as a function of stimulation phase. The letters " $a$ " and " $b$ " indicate the phase values corresponding to examples shown in $\boldsymbol{a}$ and $\boldsymbol{b}$. Middle bottom, The change in synaptic weights associated with the trained sequence as a function of stimulation phase. Bottom, The probability of Up state initiation at Group A (neuron \#50-54) as a function of stimulation phase. Error bars indicate SEM. Gray line indicates the value from uncued sleep. Blue and red regions represent Down and Up states of the SO, respectively.

recent experimental data that revealed that the learning-related cues would preferentially strengthen associated memories when they are delivered at the Down phase of SO (Batterink et al., 2016). Our study proposes a possible mechanism for such phasedependent reorganization of the structure of the slow waves to promote specific sequence replay, and provides insight into how the memory consolidation may be affected by sensory cues applied during sleep SOs.

\section{Closed-loop stimulation enhances SO power and peak frequency}

To compare model predictions with experimental data (Ngo et al., 2013, 2015), we next tested performance of the closed-loop 2-Click protocol. Above, we found that the optimal phase for stimulation was when the cues were delivered $\sim 500 \mathrm{~ms}$ after detecting the onset of a Down state. In the Best Phase protocol, stimulation was delivered at the optimal phase at each cycle of SO. The 2-Click protocol consists of 2 subsequent clicks delivered at the optimal phase with a pause of $2.5 \mathrm{~s}$ after the second click (Fig. 5a) to match the experimental protocol of Ngo et al. (2015). In the Sham (No stimulation) condition, no cue was presented during sleep. Compared with the Sham condition, the cue presentation at Best Phase significantly increased SO power (Fig. $5 b$; $p=3.18 \times 10^{-13}$, pairwise comparison), shifted the peak frequency in the SO range $(0.2-1 \mathrm{~Hz})$ toward higher frequencies (Fig. $5 c ; p=1.3 \times 10^{-11}$, pairwise comparison), and increased the performance of sequence recall after sleep (Fig. $5 d ; p=0.001$, pairwise comparison). These results are all consistent with experimental data (Fig. 5e-g, modified from Ngo et al., 2015). Although we found slight differences in the SO power and peak frequency (Fig. $5 b, p=0.0324$; Fig. $5 c, p=0.0104$; pairwise comparison), there was no significant difference in performance between Best Phase and 2-Click stimulation protocols after sleep (Fig. $5 d ; p=0.0773$, pairwise comparison). Importantly, this result is consistent with experimental data (Fig. $5 e-g$, modified from Ngo et al., 2015).

We previously reported that the spatiotemporal pattern of slow waves has a "history effect"; after slow wave is initiated at one network site, there is a higher probability for initiation to occur again at the same site, and this effects lasts for 3 or 4 subsequent cycles of SO (Wei et al., 2016). We believe this phenomenon can explain the results of our new stimulation experiments. Indeed, when stimulation skips only 1 or 2 cycles of SO (as in 2-Click protocol), during these cycles, the network still has higher than chance probability for new Up state initiation near the last initiation site. This prediction is supported by analysis in Figure $5 \mathrm{~h}$ where we plot the distribution of the Up state initiation sites across the network for Best Phase (Fig. 5h, left) and 2-Click (Fig. $5 h$, middle) stimulation protocols, and we compared them with the Sham case. We found that the first two distributions (Fig. 5h, left and middle, vertical histograms) are very similar and show peaks at the stimulation location, whereas the third distribution (Fig. 5h, right, vertical histograms) does not have any clear peak. Thus, for 2-Click stimulation protocol, the network dynamics is 


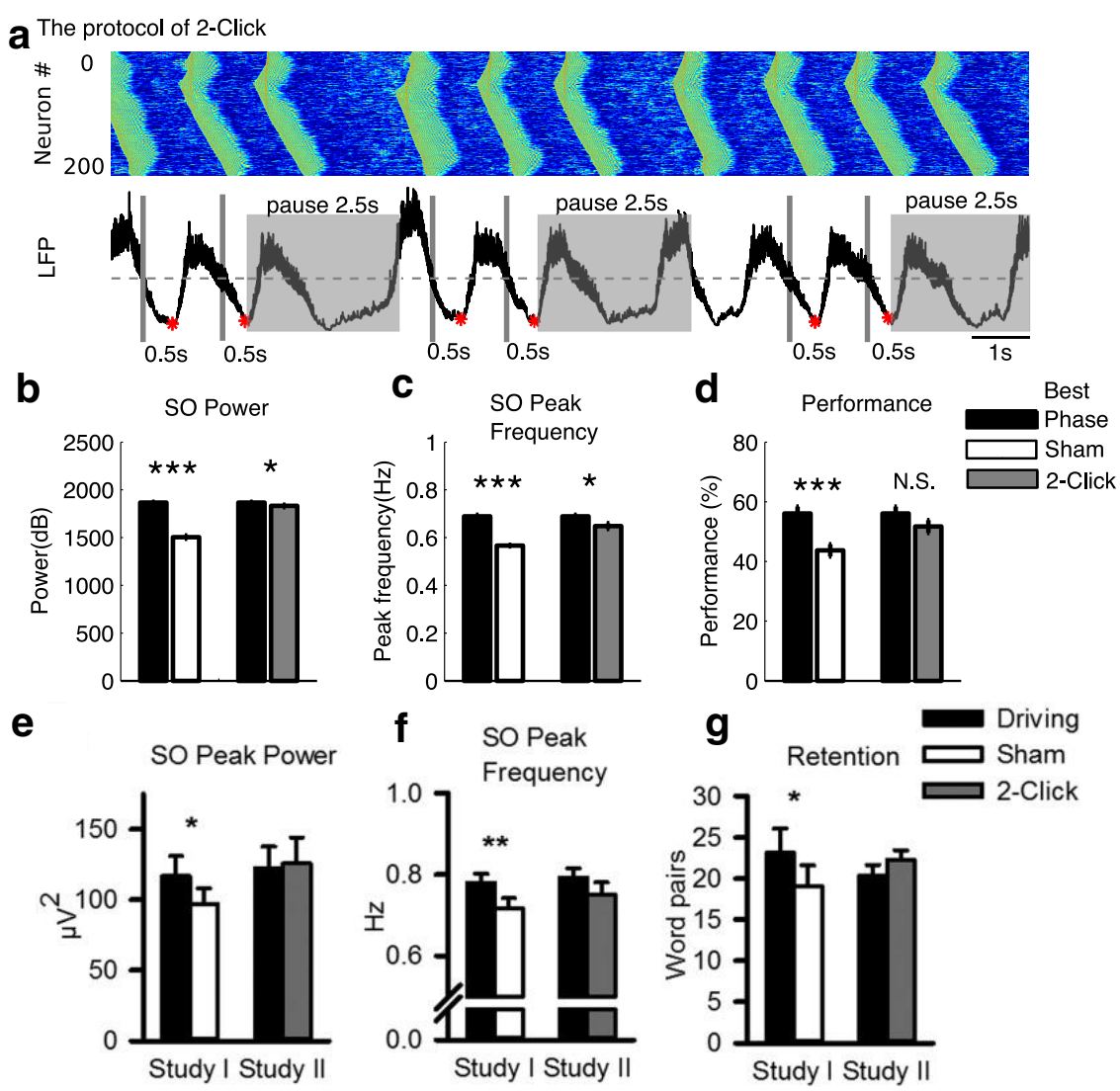

h
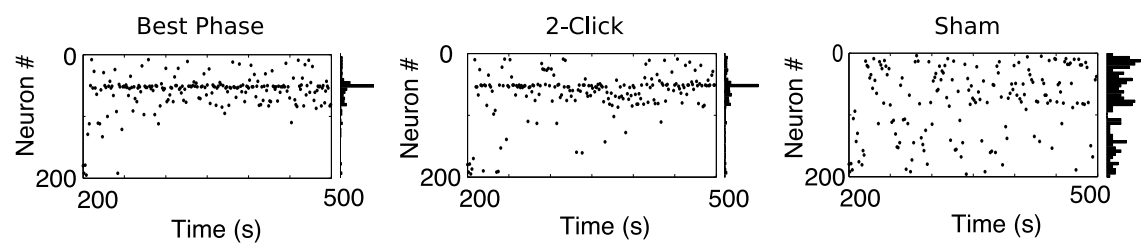

Figure 5. Closed-loop stimulation enhances SO power, peak frequency, and performance. $\boldsymbol{a}$, The protocol is based on two subsequent stimuli: 2 -Click. The cues were triggered $0.5 \mathrm{~s}$ after detecting the onset of a Down state. The detection routine was paused for $2.5 \mathrm{~s}$ after the second cue presentation. Red star indicates the time when the cue was presented. $\boldsymbol{b}-\boldsymbol{d}$, SO power, SO peak frequency, and performance after sleep are compared between Best Phase and Sham, as well as between Best Phase and 2-Click. The stimulation protocol for Best Phase was the same as for 2-Click, but stimuli were presented at each Up state without pausing the detection routine. For the Sham condition, time points of stimulation were marked, but no actual cue was presented. $\boldsymbol{e}-\boldsymbol{g}$, S0 peak power, S0 peak frequency, and retention from experimental data (modified from Ngo et al., 2015). $\boldsymbol{h}$, Black dots represent Up state initiation sites over the sleep period during Best Phase (left), 2-Click (middle), and Sham (right) stimulation protocols. Right, Vertical panels represent probability of Up state initiation across entire network. ${ }^{*} p<0.05 .{ }^{* *} p<0.01$. ${ }^{* * *} p<$ 0.001. N.S. represents no significance.

effectively identical to that during continuous stimulation (Best Phase), and this explains the nearly identical performance.

\section{Closed-loop stimulation protocol with the entire} network activation

From the perspective of the cortical neurons, it remains unclear how focal or distributed the effect of the sensory stimulation is during sleep. In the next set of model stimulations, instead of the input targeting only a single Group A of five neurons, stimulation targeted a much larger neuronal population (Fig. $6 a$, left top). The phase of closed-loop stimulation was selected near the end of a Down state, before the onset of an Up state (Fig. 6a, right), based on the results we obtained in the previous modeling experiments. We found that the amplitude of SO (Fig. $6 a$, left middle) was significantly increased in such stimulation conditions due to the very synchronized network transitions from Down to Up states (Fig. $6 a$, left). Up state initiation front was also broader (more neurons transited to Up states within relatively small time window) but still was centered near the trained population of neurons (Fig. $6 a$, left bottom). In another experiment (Fig. 6b), the broad stimulation cue was presented $200 \mathrm{~ms}$ after the onset of the Up state (Fig. $6 b$, left middle, red star). At that phase of stimulation, the large fraction of the cortical neuron population was in the Up state already (phase $\sim 330^{\circ}$ ) (Fig. $6 b$, right); therefore, stimulation had minimal impact on the location of the Up state initiation site and slow-wave spatiotemporal pattern (Fig. 6b, left bottom). Thus, when the broad cue was presented during the Down state $\left(90^{\circ}-270^{\circ}\right)$ of SOs, the performance (Fig. $6 c$, middle top), the synaptic weight change associated with the sequence (Fig. $6 c$, middle bottom), and the probability of Up state initiation at Group A of neurons (Fig. $6 c$, bottom) were all higher than those when the cue was presented during the Up state $\left(270^{\circ}-360^{\circ}\right.$ and $0^{\circ}-90^{\circ}$ ), and also higher than those in the uncued model (Fig. $6 c$, gray lines). These findings are similar to what we observed during the local cue presentation.

To quantify how external stimulation affects the spatiotemporal pattern of SO, we measured the PLI of focal LFPs during individual Up states (Fig. $7 a$; each line indicates the population activity from 25 neurons; we excluded the boundary neurons from analysis). The following three conditions were compared (Fig. 7b): Sham $(\mathrm{PLI}=0.4826 \pm 0.0306)$, cueing a local group of neurons (local cue) at the optimal stimulation phase (PLI = $0.6387 \pm 0297)$, and cueing a broad group of neurons (broad cue) at the optimal stimulation phase $(\mathrm{PLI}=0.5793 \pm$ 0.0166). We found that PLI for the Sham group was significantly lower than for the model using local stimulation at site $\mathrm{A}$ only $\left(p=1.72 \times 10^{-25}\right.$, one-way ANOVA Bonferroni corrections) as well as for the model using broad stimulation ( $p=$ $4.06 \times 10^{-16}$, one-way ANOVA Bonferroni corrections).

We next calculated cross-correlations between all possible LFP pairs and plotted the distribution of the time lags to the peak (Fig. $7 c$ ). In the Sham condition, the distribution was relatively broad with multiple local peaks (Fig. $7 c$, left), suggesting that many different network sites could lead to transitions from Down to Up states at different SO cycles or even during any one cycle of SO. When the local stimulation was applied at the optimal phase, the lags peaked $\sim-100 \mathrm{~ms}$ (Fig. $7 c$, middle), suggesting the pattern of propagation of the slow waves from one preferential location, which can be explained by the locally stimulated region being the main initiation site. When the stimulation was applied across a broad region at the optimal phase, the lags peaked $\sim 0 \mathrm{~ms}$ 
(Fig. $7 c$, right), indicating that the broad stimulation increased network synchronization with zero lag.

\section{Selective stimulation targets specific memories during sleep replay}

To test how the targeted stimulation during sleep affects only one memory when several memories are trained, we trained two independent sequences at two different network locations (Fig. 8a). We used long enough initial training time for both sequences to ensure that they replay and consolidate during SWS regardless of the interference effects (Wei et al., 2018). In the model, two sequences were trained by sequentially presenting stimuli at Group $\mathrm{A}_{1}$ (\#50-54), $\mathrm{B}_{1}$ (\#55-59), $\mathrm{C}_{1}$ (\#60-64), $\mathrm{D}_{1}$ (\#65-69), $\mathrm{E}_{1}$ (\#70-74) for Seq1, and at Group $E_{2}$ (\#146-150), $D_{2}$ (\#141-145), $C_{2}$ (\#136-140), $\mathrm{B}_{2}$ (\#131-135), $\mathrm{A}_{2}$ (\#126130) for Seq2, respectively (Fig. $8 b$, left). Each sequence was paired with a different sensory cue. During test sessions, as before, the recall performance for each sequence was measured based on the network response by stimulating only the first group of neurons in each sequence: Group $\mathrm{A}_{1}$ or Group $\mathrm{E}_{2}$ (Fig. $8 b$, right). When two sequences were trained for the same duration of $80 \mathrm{~s}$, and no cues were presented during sleep, recall performance was similar for both tasks (Fig. $8 c, d$, left, Uncued). Specifically, the performance for recalling Seq1 before training, after training before sleep, and after sleep was $17.9 \pm 1.37 \%, 27.4 \pm 1.43 \%$, and $44.7 \pm 2.02 \%$, respectively (Fig. $8 c$, top), whereas the performance for recalling Seq 2 before training, after training before sleep, and after sleep was $17.5 \pm$ $0.96 \%, 32.6 \pm 1.72 \%$, and $49.8 \pm 2.46 \%$, respectively (Fig. $8 c$, bottom). For Seq1, the performance was significantly increased after training compared with before training $\left(p=3.78 \times 10^{-4}\right.$, one-way ANOVA, Bonferroni corrections), and further significantly improved after sleep compared with before sleep after training $\left(p=1.44 \times 10^{-9}\right.$, one-way ANOVA, Bonferroni corrections). For Seq2, the performance was significantly increased after training compared with before training $\left(p=6.94 \times 10^{-7}\right.$, one-way ANOVA, Bonferroni corrections), and further significantly improved after sleep compared with before sleep after training $(p=$ $3.12 \times 10^{-8}$, one-way ANOVA, Bonferroni corrections).

While both sequences revealed similar performances when they were trained for the same duration without presenting any cues during sleep, results changed when a The broad cue applied 500ms after the onset of Down state

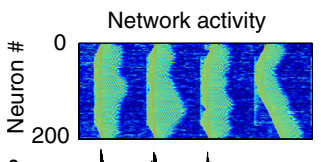
Circular histogram of phase
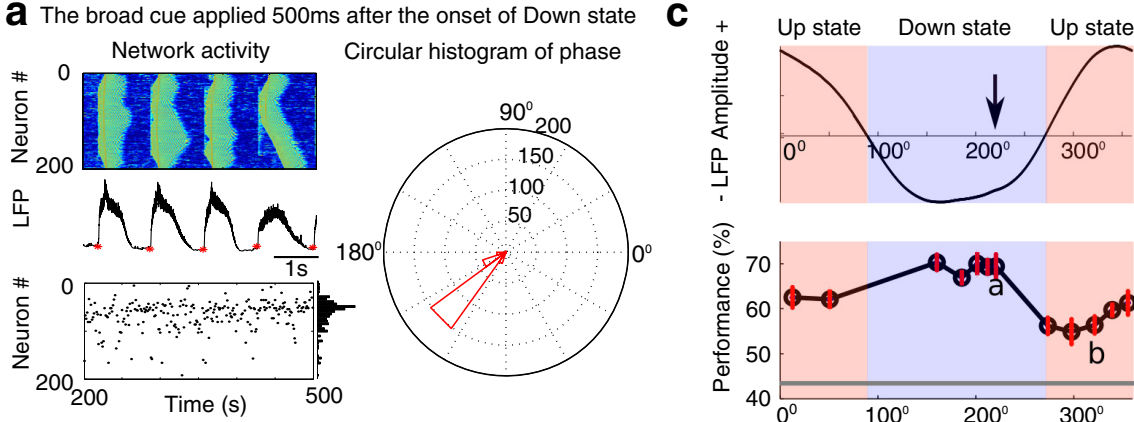

b The broad cue applied 200ms after the onset of Up state

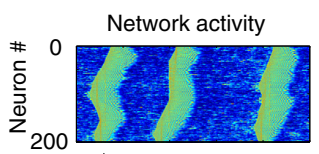
Circular histogram of phase
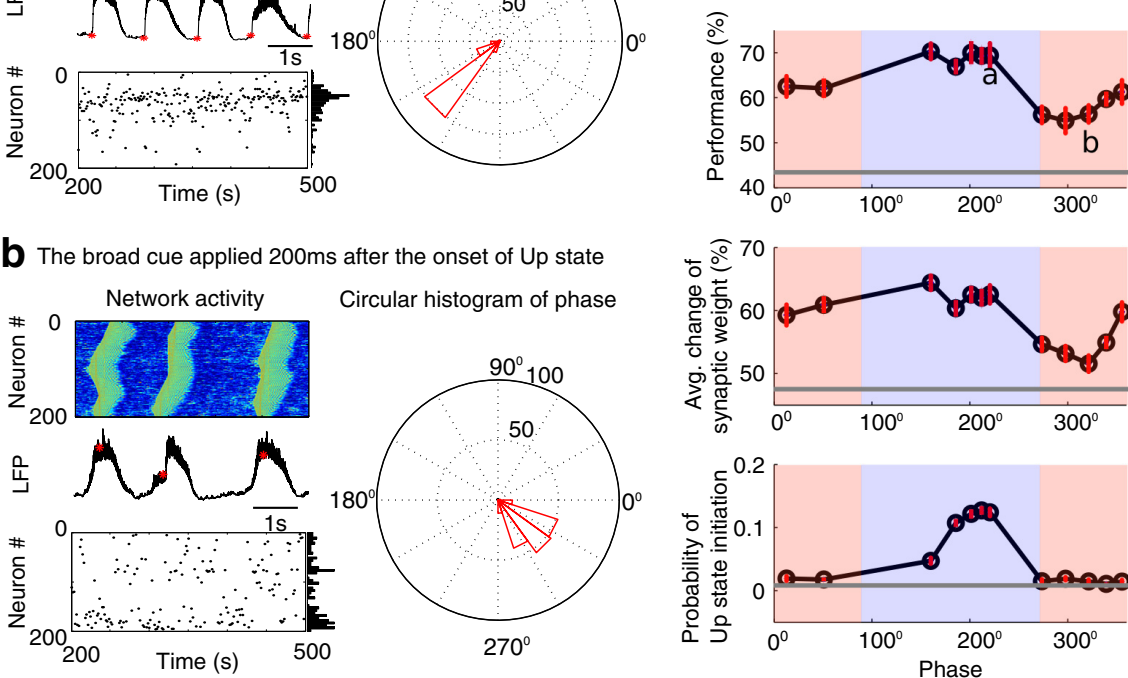

Figure 6. Closed-loop stimulation protocol with a broad cue input during slow oscillations. $\boldsymbol{a}, \boldsymbol{b}$, Examples of cue presentation for two different phases of S0. Cue presentations in $\boldsymbol{a}$ and $\boldsymbol{b}$ correspond to $500 \mathrm{~ms}$ after the Up to Down state transition and $200 \mathrm{~ms}$ after the Down to Up state transition, respectively. $\boldsymbol{a}, \boldsymbol{b}$, Top left, Characteristic example of network activity during SOs. Middle left, Characteristic example of LFP. Red stars represent the times of stimulation. Bottom left, Black dots represent Up state initiation sites during the entire sleep period. Right, Vertical panel represents probability of Up state initiation across neurons. Right, Circular histogram of phases at which the cue was applied. c, Top, The correspondence between phase angle and states of SO (Up and Down states). Vertical arrow indicates the optimal phase for cue presentation. Middle top, The performance after sleep as a function of stimulation phase. The letters " $a$ " and " $b$ " indicate the phase values shown in $\boldsymbol{a}$ and $\boldsymbol{b}$. Middle bottom, The change of synaptic weights associated with trained sequence as a function of stimulation phase. Bottom, The probability of Up state initiation at Group A (neuron \#50-54) as a function of stimulation phase. Error bars indicate SEM. Gray line indicates the value from uncued sleep. Blue and red regions represent Down and Up states of the $S 0$, respectively.

A Example of focal LFP during one Up state

b Phase locking index for different conditions
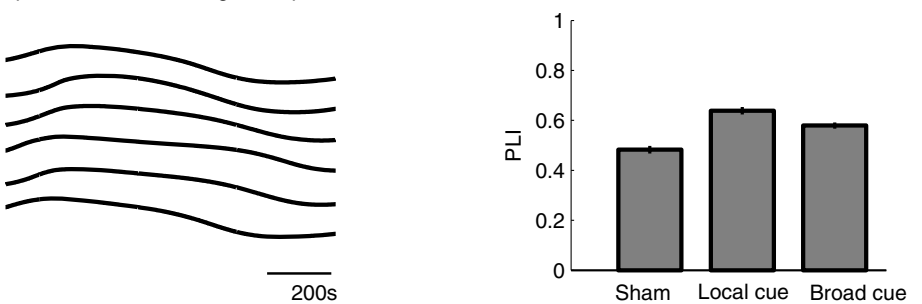

The histogram of lags at peak cross correlation Sham
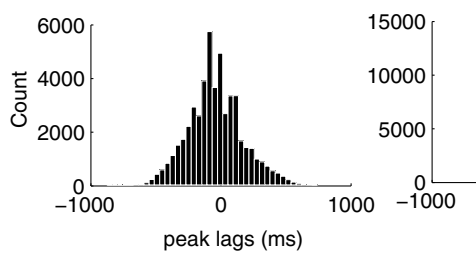

Local cue

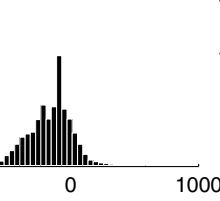

peak lags (ms)
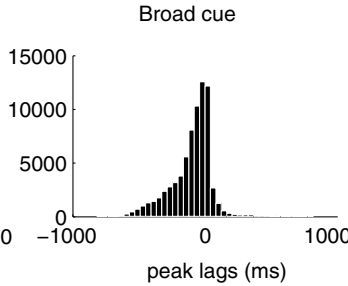

Figure 7. The spatial pattern of sleep $\mathrm{S} 0$ in three different conditions: Sham, local cue, and broad cue. $\boldsymbol{a}$, Example of population activity (every 25 neurons) during one characteristic Up state. The boundary neurons are not included. $\boldsymbol{b}, \mathrm{PLI}$ for different conditions: Sham, local cue, and cue. Local and broad cues were presented at the optimal phase. The local cue was targeted only at Group A, whereas the broad cue was targeted at all the neurons, excluding the boundary neurons. c, Histograms of lags at peak crosscorrelation for all three conditions. 
a Sequence learning framework

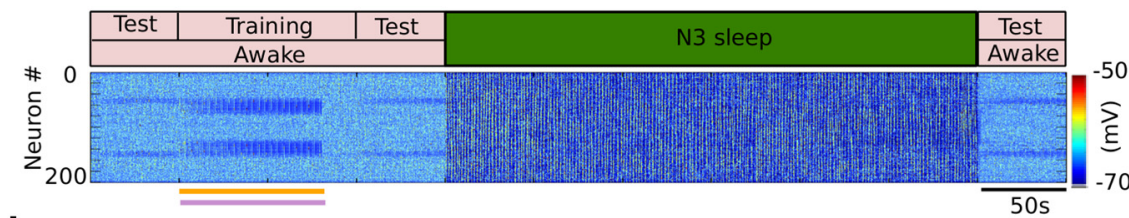

b

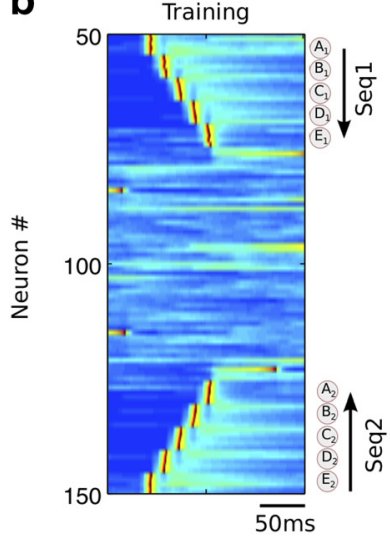

Test

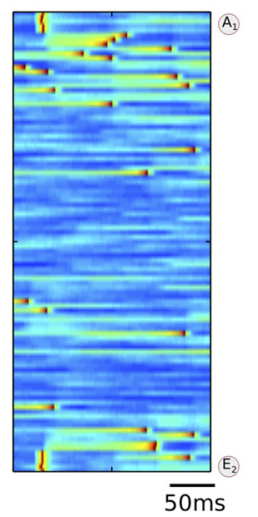

C
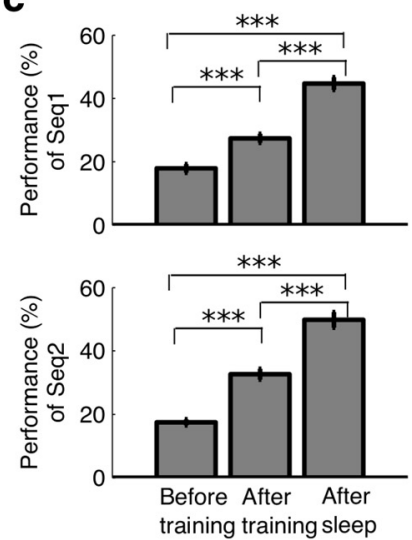

d The performance improvement after sleep compared with before sleep
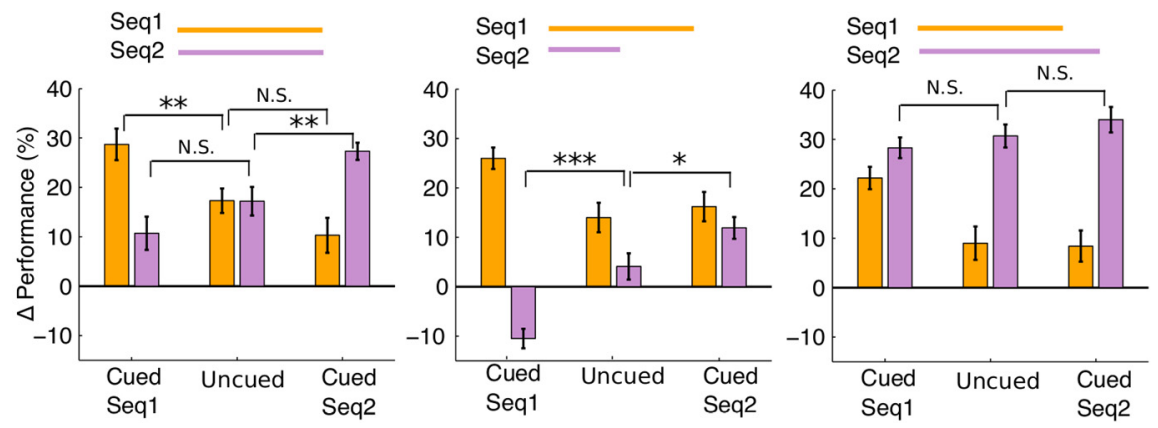

Figure 8. The cue can selectively enhance the specific memory. $\boldsymbol{a}$, The model of transitions between awake and N3 sleep (SWS). Two spike sequences were trained during awake. Orange and purple bars represent the duration of training for Seq1 and Seq2, respectively. $\boldsymbol{b}$, Characteristic example of training and testing of Seq1 $\left(A_{1}, B_{1}, C_{1}, D_{1}, E_{1}\right)$ and $\operatorname{Seq} 2\left(E_{2}, D_{2}, C_{2}, B_{2}, A_{2}\right)$. The test was applied by stimulating only Group $A_{1}$ for Seq1 and Group $E_{2}$ for Seq2. Seq1 and Seq2 started at neuron \#50 and \#150, respectively. c, Bar plots of performance for Seq1 and Seq2 during test sessions. Error bars indicate SEM. $\boldsymbol{d}$, Performance improvement due to sleep ( $\Delta$ performance) for Seq1 (orange) and Seq2 (purple) in three cases: (1) Seq2 was trained for the same duration as Seq1 (left); (2) Seq2 was trained less than Seq1 (middle); and (3) Seq2 was trained more than Seq1 (right). In each condition, we show results of three experiments: (1) Uncued — none of the sequences were cued during sleep; (2) Cued Seq1 — only the cue associated with Seq1 was presented during sleep; and (3) Cued Seq2 — only the cue associated with Seq2 was presented during sleep. ${ }^{*} p<0.05$. ${ }^{* *} p<0.01 .{ }^{* * *} p<0.001$. N.S. represents no significance.

the sensory cue was applied during sleep (Fig. $8 d$, left). Here, we defined $\Delta P$ as the performance after sleep minus the performance before sleep, indicating the performance improvement due to sleep. If only the cue associated with Seq1 was presented during sleep, $\Delta P$ for Seq1 was significantly increased (Uncued vs Cued Seq1: $17.3 \pm 2.48 \%$ vs $28.7 \pm 3.20 \%, t_{(38)}=2.877, p=0.0076$, two-sample $t$ test; Fig. $8 d$, left, orange bar), whereas $\Delta P$ for Seq 2 was reduced, but not significantly (Uncued vs Cued Seq1: $17.2 \pm$ $2.90 \%$ vs $10.7 \pm 3.38 \%, t_{(38)}=-1.4614, p=0.1521$, two-sample $t$ test; Fig. $8 d$, left, purple bar). If only the cue associated with Seq2 was presented during sleep, $\Delta P$ for Seq2 was significantly increased (Uncued vs Cued Seq2: $17.2 \pm 2.90 \%$ vs $27.3 \pm 1.74 \%$, $t_{(38)}=-2.9881, p=0.0049$, two-sample $t$ test; Fig. $8 d$, left, purple bar), whereas $\Delta P$ for Seq1 was reduced, but not significantly (Uncued vs Cued Seq2: $17.3 \pm 2.48 \%$ vs $0.3 \pm 3.53 \%, t_{(38)}=1.624$, $p=0.1127$, two-sample $t$ test; Fig. $8 d$, left, orange bar). These results suggest that presenting the cue associated with one specific memory sequence accelerates the consolidation of that sequence without significantly interfering with the consolidation of another memory sequence.

We next used different training times for two sequences: Seq2 was trained less than Seq1 (Fig. 8d, middle). This difference (Seq2 has higher performance than Seq1) remained after sleep in uncued condition (Fig. 8d, middle, Uncued). In such case, when the cue associated with Seq1 was presented during sleep, $\Delta P$ for Seq 2 was significantly reduced compared with the uncued condition (Cued Seq1 vs Uncued: $-10.5 \pm 1.97 \%$ vs $4.1 \pm 2.66 \%, t_{(38)}$ $=-4.4112, p=8.19 \times 10^{-5}$, two-sample $t$ test; Fig. $8 d$, middle, purple bar) and even became negative, indicating that weaker memory of Seq2 was partially erased due to the strong reactivation of Seq1. When the cue associated with weaker Seq 2 was presented, $\Delta P$ for Seq 2 was significantly increased (Uncued vs Cued Seq2: $4.1 \pm 2.66 \%$ vs $11.9 \pm 2.21 \%$, $t_{(38)}=-2.2537, p=0.03$, two-sample $t$ test; Fig. $8 d$, middle, purple bar), without significant impact on Seq 1 performance.

Last, we trained Seq2 longer than Seq1; therefore, the initial (before sleep) memory strength of Seq2 was higher than that of Seq1 (Fig. $8 d$, right). After sleep, in uncued condition, Seq 2 showed a very significant performance increase, whereas Seq 1 improvement was relatively reduced (compare Fig. $8 d$, right, Uncued to Fig. $8 d$, left, Uncued) likely because of the interference between two sequences during sleep replay (Wei et al., 2018). If only the cue associated with Seq1 was presented, $\Delta P$ for Seq1 was increased significantly, but $\Delta P$ for Seq 2 was similar to that after uncued sleep. If only the cue associated with Seq 2 was presented, $\Delta P$ for both Seq 1 and Seq 2 was also not significantly different compared with uncued sleep (Uncued vs Cued Seq2: $30.7 \pm 2.33 \%$ vs $34 \pm$ $2.58 \%, t_{(38)}=-0.9483, p=0.349$, two-sample $t$ test, Fig. $8 d$, right, purple bar), indicating that consolidation of the stronger Seq2 does not have room to further increase in the presence of a cue. Overall, these results suggest the following: (1) cues during SWS are particularly effective to improve consolidation of weak memories; and (2) applying cues associated with one specific memory may lead to performance degradation for other, particularly weak memories, that are not cued.

\section{Discussion}

In this study, using a realistic computational model of the thalamocortical network implementing sleep stages (Krishnan et al., 2016) and synaptic plasticity (Wei et al., 2016, 2018), we aimed at developing a better understanding of how exogenous stimulation during sleep can enhance memory consolidation. Our study suggests that both training-associated cues or auditory stimulation during SWS can promote spike sequence replay and enhance memory performance after sleep. The cues only 
strengthened the memory traces when delivered at or near the optimal phase, just before the Down to Up state transition of the slow waves. The same phase relationship also applied for auditory stimulation, not previously related to the learning context. In both cases, consolidation was improved because stimulation was able to shape the spatiotemporal pattern of sleep slow waves to promote Up state initiation near the site when trained memory was encoded. When multiple memories were trained and one memory was associated with a specific cue during training, sensory cues during SWS could selectively strengthen the associated memories but could also lead to performance degradation for other, particularly weak, memories.

\section{The mechanisms of strengthening memories by stimulation during NREM sleep}

Synaptic plasticity is believed to be the cellular mechanism of learning and memory in the brain (Ho et al., 2011). Evidence is accumulating that recent memories are consolidated during NREM sleep (Walker and Stickgold, 2004; Diekelmann and Born, 2010; Born and Wilhelm, 2012; Rasch and Born, 2013) through replay of sequences of cell-firing patterns that occur during waking (Euston et al., 2007; Ji and Wilson, 2007; Peyrache et al., 2009). This replay is thought to be orchestrated by hippocampal and thalamocortical patterns of activity (Marshall et al., 2006; Girardeau et al., 2009; Ego-Stengel and Wilson, 2010; Ngo et al., 2013; Maingret et al., 2016). We recently showed, using computer models, that sequences of cortical neurons trained in awake are replayed spontaneously during NREM sleep; this enhances the synaptic connections associated with the trained memory resulting in memory improvement (Wei et al., 2018). Furthermore, sleep replay during SWS is able to protect old memories from catastrophic forgetting associated with new learning (González et al., 2019; Krishnan et al., 2019). In this present study, we explored how external stimulation augments an internal and naturally occurring mechanism of memory reactivation, resulting in an enhanced memory consolidation process.

Recently, empirical studies revealed a powerful new tool to augment the memory consolidation process: TMR during sleep (for review, see Oudiette and Paller, 2013; Schouten et al., 2017). In these experiments, the stimuli (e.g., sounds or odors) presumably enhance reactivation of the relevant neuronal representations that improve memory consolidation. TMR was shown to improve both hippocampus-independent procedural memories (Antony et al., 2012; Cousins et al., 2014, 2016; Schönauer et al., 2014) and hippocampus-dependent declarative memories (Rasch et al., 2007; Rudoy et al., 2009; Batterink et al., 2016; Oyarzun et al., 2017). In one experiment, an odor was associated with learning a spatial card location task; subsequent presentation of the odor cue during sleep led to selective enhancement of this memory compared with the sleep without cueing (Rasch et al., 2007). Similarly, auditory cues that were paired with visual input during learning enhanced memory consolidation when presented during subsequent sleep (Rudoy et al., 2009). The timing of the stimulation with respect to ongoing sleep SO determined the effect of a cue on memory consolidation (Batterink et al., 2016; Santostasi et al., 2016). Specifically, the closed-loop stimulation presented during the Down state, just before transition from the Down to Up state of SOs, had high impact on memory consolidation compared with other stimulation phases. For overlapping memories, whether the auditory cues strengthen or weaken a memory also depended on the memory strength (Oyarzun et al., 2017). Notably, the beneficial effect of external reactivation only occurred during sleep. Presenting the same cues during wakefulness was not effective for either declarative memories (Rasch et al., 2007) or procedural memories (Schönauer et al., 2014).

We recently reported, using biophysical models of the thalamocortical system, that sleep reactivation occurs during Up states of the SO (Wei et al., 2018). When the synaptic connectivity matrix was modified in the awake state by sequence training, the trained sequences were spontaneously replayed during Down to Up state transitions and also during early phases of the Up state, leading to further changes in synaptic weights (consolidation). For memories represented by the sequences of neuronal activation (as in this study), the beginning of a sequence was commonly also a site where local spontaneous Up states were initiated. In this new study, we showed that the effect of stimulation on memory replay depends on the ability of stimuli to affect, at least locally, the spatiotemporal pattern of sleep slow waves. When a local stimulus was delivered at the "correct" phase of the SO (during Down states but close to the transition to Up states) and to the "correct" network location (near the trained sequence), it effectively triggered local Up state initiation and promoted replay of a sequence associated with this stimulation site. Stimuli delivered at "wrong" timing (e.g., when the network was already active or just transited to the Down state) or "wrong" location (e.g., far away from the beginning of a sequence in question) could not facilitate replay of that sequence.

\section{Mechanisms of sequence replay}

Synaptic plasticity is believed to be the cellular mechanism of learning and memory in the brain. A large body of studies supports the idea that the spike sequences of cortical neurons evoked by awake learning are spontaneously replayed during sleep, leading to consolidation of memory (Euston et al., 2007; Ji and Wilson, 2007; Peyrache et al., 2009; Barnes and Wilson, 2014; Ramanathan et al., 2015). Presently, many studies showed that stimulation, such as training-associated cues (Rasch et al., 2007; Rudoy et al., 2009; Antony et al., 2012; Cousins et al., 2014, 2016; Schönauer et al., 2014), or auditory stimulation (Ngo et al., 2013, 2015), presented during sleep can augment consolidation of the newly encoded memories.

The nature of performance improvement in the model presented in this new study is linked to the effect of STDP on synaptic weights during sequential spiking. Initial training led to sequential activation in groups of neurons (e.g., $A \rightarrow B \rightarrow C \rightarrow D \rightarrow E$ ), which created asymmetric weight configurations within trained regions where all the weights in the direction of sequence training (e.g., $\mathrm{A} \rightarrow \mathrm{B}$ ) increased and the weights in the opposite direction (e.g., B $\rightarrow$ A) decreased. During SWS, local waves traveling in direction of sequence training further increased this synaptic weight asymmetry; therefore, performance increased after sleep. This effect extended beyond initial wave front propagation as the neurons largely preserved "correct" timing within the entire Up state. However, in the Sham condition, without external stimulation, many slow waves were initiated randomly, and this limited replay. In the model simulating sensory stimulation, input from the sensory network increased the likelihood of the local Up state activation at the network site near the beginning of the sequence location (e.g., A). Effectively, it increased replay and led to performance improvement after sleep with cues.

\section{Closed-loop auditory stimulation during sleep: local or broad cue?}

Different types of auditory stimulation, represented either by the learning-specific cues (Batterink et al., 2016) or pink noise (Ngo 
et al., 2013, 2015; Santostasi et al., 2016), are all able to enhance memory consolidation. We found, however, that the optimal phase and the mechanisms were somewhat different for these two different types of sensory stimulation.

For the learning-specific cue, we hypothesized that the cue only affects a small population of neurons (local cue). This may explain why stimulation is only effective in vivo when it was applied at specific phase of the slow waves (Batterink et al., 2016). In our study, we identified that the optimal phase depends on the influence of the stimuli on the Up state initiation. When stimulus is delivered just before Down to Up state transitions when the network is sensitive to external perturbations, the stimulus affects (possibly locally) the spatiotemporal pattern of slow waves promoting the sequence replay and increase in synaptic weights associated with the trained sequence in question.

For the pink noise auditory stimulation, stimulation can potentially activate a broad group of neurons (broad cue). It was shown in vivo that such broad stimuli could enhance memory consolidation by enhancing the SO (Ngo et al., 2013, 2015). Importantly, the pink auditory stimulation was effective in vivo when the sound occurred in synchrony with SO Up states (Ngo et al., 2013). In the model, broader stimulation led to a higher increase in memory performance compared with the local one. Also, while the peak performance was still achieved for stimuli delivered at the network transition from Down to Up states, the difference was smaller between optimal and suboptimal stimulations. In all cases, an increase in performance was linked to a significant increase in amplitude and synchrony of SOs, in agreement with in vivo data. Considering that in vivo the SO is not necessarily well synchronized across the entire cortex (and definitely less synchronized than that in our model) (Nir et al., 2011), we predict that, for broader stimuli, the optimal stimulation would be during the early phase of an Up state when it is most capable of inducing high-amplitude synchronized slow wave across large populations of the cortical neurons.

Together, our study provides insight into how memory consolidation may be affected by stimulation applied during SWS. We consider stimulation both as a tool to manipulate the sleep rhythms to understand mechanisms behind the role of sleep in memory consolidation and as an approach to develop clinical interventions with a goal of enhancing memory and learning. The auditory stimulation during sleep has the potential application to enhance sleep-dependent effects on memory in cognitively healthy human subjects and in those with amnestic mild cognitive impairment.

\section{References}

Andrillon T, Nir Y, Staba RJ, Ferrarelli F, Cirelli C, Tononi G, Fried I (2011) Sleep spindles in humans: insights from intracranial EEG and unit recordings. J Neurosci 31:17821-17834.

Antony JW, Gobel EW, O'Hare JK, Reber PJ, Paller KA (2012) Cued memory reactivation during sleep influences skill learning. Nat Neurosci 15: $1114-1116$

Barnes DC, Wilson DA (2014) Slow-wave sleep-imposed replay modulates both strength and precision of memory. J Neurosci 34:5134-5142.

Batterink LJ, Creery JD, Paller KA (2016) Phase of spontaneous slow oscillations during sleep influences memory-related processing of auditory cues. J Neurosci 36:1401-1409.

Bazhenov M, Timofeev I, Steriade M, Sejnowski TJ (2002) Model of thalamocortical slow-wave sleep oscillations and transitions to activated states. J Neurosci 22:8691-8704.

Blake H, Gerard RW (1937) Brain potentials during sleep. Am J Physiol 119:692-703.

Born J, Wilhelm I (2012) System consolidation of memory during sleep. Psychol Res 76:192-203.
Chauvette S, Seigneur J, Timofeev I (2012) Sleep oscillations in the thalamocortical system induce long-term neuronal plasticity. Neuron 75:11051113.

Chen JY, Chauvette S, Skorheim S, Timofeev I, Bazhenov M (2012) Interneuron-mediated inhibition synchronizes neuronal activity during slow oscillation. J Physiol 590:3987-4010.

Cousins JN, El-Deredy W, Parkes LM, Hennies N, Lewis PA (2014) Cued memory reactivation during slow-wave sleep promotes explicit knowledge of a motor sequence. J Neurosci 48:15870-15876.

Cousins JN, El-Deredy W, Parkes LM, Hennies N, Lewis PA (2016) Cued reactivation of motor learning during sleep leads to overnight changes in functional brain activity and connectivity. PLoS Biol 14:e1002451.

Destexhe A, Bal T, McCormick DA, Sejnowski TJ (1996) Ionic mechanisms underlying synchronized oscillations and propagating waves in a model of ferret thalamic slices. J Neurophysiol 76:2049-2070.

Diekelmann S, Born J (2010) The memory function of sleep. Nat Rev Neurosci 11:114-126.

Ego-Stengel V, Wilson MA (2010) Disruption of ripple-associated hippocampal activity during rest impairs spatial learning in the rat. Hippocampus 20:1-10.

Euston DR, Tatsuno M, McNaughton BL (2007) Fast-forward playback of recent memory sequences in prefrontal cortex during sleep. Science 318: $1147-1150$

Girardeau G, Benchenane K, Wiener SI, Buzsáki G, Zugaro MB (2009) Selective suppression of hippocampal ripples impairs spatial memory. Nat Neurosci 12:1222-1223.

González OC, Sokolov Y, Krishnan GP, Bazhenov M (2019) Can sleep protect memories from catastrophic forgetting? bioRxiv 569038. doi:https:// doi.org/10.1101/569038.

Ho VM, Lee JA, Martin KC (2011) The cell biology of synaptic plasticity. Science 334:623-628.

Ji D, Wilson MA (2007) Coordinated memory replay in the visual cortex and hippocampus during sleep. Nat Neurosci 10:100-107.

Kimura F, Fukuda M, Tsumoto T (1999) Acetylcholine suppresses the spread of excitation in the visual cortex revealed by optical recording: possible differential effect depending on the source of input. Eur J Neurosci 11:3597-3609.

Krishnan GP, Chauvette S, Shamie I, Soltani S, Timofeev I, Cash SS, Halgren E, Bazhenov M (2016) Cellular and neurochemical basis of sleep stages in the thalamocortical network. eLife 5:e18607.

Krishnan GP, Tadros T, Ramyaa R, Bazhenov M (2019) Biologically inspired sleep algorithm for artificial neural networks. arXiv:1908.02240.

Kuznetsov YA (1995) Elements of applied bifurcation theory. In: Applied mathematical sciences. New York: Springer.

Loomis AL, Harvey EN, Hobart G (1935) Potential rhythms of the cerebral cortex during sleep. Science 81:597-598.

Mainen ZF, Sejnowski TJ (1996) Influence of dendritic structure on firing pattern in model neocortical neurons. Nature 382:363-366.

Maingret N, Girardeau G, Todorova R, Goutierre M, Zugaro M (2016) Hippocampo-cortical coupling mediates memory consolidation during sleep. Nat Neurosci 19:959-964.

Marshall L, Helgadóttir H, Mölle M, Born J (2006) Boosting slow oscillations during sleep potentiates memory. Nature 444:610-613.

McCormick DA (1992) Neurotransmitter actions in the thalamus and cerebral cortex and their role in neuromodulation of thalamocortical activity. Prog Neurobiol 39:337-388.

McCormick DA, Williamson A (1991) Modulation of neuronal firing mode in cat and guinea pig LGNd by histamine: possible cellular mechanisms of histaminergic control of arousal. J Neurosci 11:3188-3199.

Ngo HV, Martinetz T, Born J, Mölle M (2013) Auditory closed-loop stimulation of the sleep slow oscillation enhances memory. Neuron 78: 545-553.

Ngo HV, Miedema A, Faude I, Martinetz T, Mölle M, Born J (2015) Driving sleep slow oscillations by auditory closed-loop stimulation: a self-limiting process. J Neurosci 35:6630-6638.

Nir Y, Staba RJ, Andrillon T, Vyazovskiy VV, Cirelli C, Fried I, Tononi G (2011) Regional slow waves and spindles in human sleep. Neuron 70: 153-169.

Oudiette D, Paller KA (2013) Upgrading the sleeping brain with targeted memory reactivation. Trends Cogn Sci 17:142-149.

Oyarzun JP, Moris J, Luque D, de Diego-Balaguer R, Fuentemilla L (2017) Targeted memory reactivation during sleep adaptively promotes the 
strengthening or weakening of overlapping memories. J Neurosci 37: $7748-7758$.

Peyrache A, Khamassi M, Benchenane K, Wiener SI, Battaglia FP (2009) Replay of rule-learning related neural patterns in the prefrontal cortex during sleep. Nat Neurosci 12:919-926.

Ramanathan DS, Gulati T, Ganguly K (2015) Sleep-dependent reactivation of ensembles in motor cortex promotes skill consolidation. PLoS Biol 13:e1002263.

Rasch B, Born J (2013) About sleep's role in memory. Physiol Rev 93: 681-766.

Rasch B, Büchel C, Gais S, Born J (2007) Odor cues during slow-wave sleep prompt declarative memory consolidation. Science 315:1426-1429.

Rudoy JD, Voss JL, Westerberg CE, Paller KA (2009) Strengthening individual memories by reactivating them during sleep. Science 326:1079.

Santostasi G, Malkani R, Riedner B, Bellesi M, Tononi G, Paller KA, Zee PC (2016) Phase-locked loop for precisely timed acoustic stimulation during sleep. J Neurosci Methods 259:101-114.

Schönauer M, Geisler T, Gais S (2014) Strengthening procedural memories by reactivation in sleep. J Cogn Neurosci 26:143-153.

Schouten DI, Pereira SI, Tops M, Louzada FM (2017) State of the art on targeted memory reactivation: sleep your way to enhanced cognition. Sleep Med Rev 32:123-131.

Sivagnanam S, Majumdar A, Yoshimoto K, Astakhov V, Bandrowski A, Martone M, Carnevale NT (2013) Introducing the neuroscience gateway. Proceedings of the 5th International Workshop on Science Gateways, Zurich, Switzerland.

Song S, Miller KD, Abbott LF (2000) Competitive Hebbian learning through spike-timing-dependent synaptic plasticity. Nat Neurosci 3:919926.

Steriade M, Nuñez A, Amzica F (1993) Intracellular analysis of relations between the slow $(<1 \mathrm{~Hz})$ neocortical oscillation and other sleep rhythms of the electroencephalogram. J Neurosci 13:3266-3283.

Steriade M, Timofeev I, Grenier F (2001) Natural waking and sleep states: a view from inside neocortical neurons. J Neurophysiol 85:1969-1985.

Stevens CF (1993) Quantal release of neurotransmitter and long-term potentiation. Cell 72:55-63.

Sugisaki E, Fukushima Y, Tsukada M, Aihara T (2015) Contribution of endogenous acetylcholine to STDP induction. In: Advances in cognitive neurodynamics, Vol IV: Proceedings of the Fourth International Conference on Cognitive Neurodynamics (Liljenström H, ed), pp 271-276. Dordrecht: Springer Netherlands.

Timofeev I, Grenier F, Bazhenov M, Sejnowski TJ, Steriade M (2000) Origin of slow cortical oscillations in deafferented cortical slabs. Cereb Cortex 10:1185-1199.

Walker MP, Stickgold R (2004) Sleep-dependent learning and memory consolidation. Neuron 44:121-133.

Wei Y, Krishnan GP, Bazhenov M (2016) Synaptic mechanisms of memory consolidation during sleep slow oscillations. J Neurosci 36:4231-4247.

Wei Y, Krishnan GP, Komarov M, Bazhenov M (2018) Differential roles of sleep spindles and sleep slow oscillations in memory consolidation. PLoS Comput Biol 14:e1006322.

Weigenand A, Mölle M, Werner F, Martinetz T, Marshall L (2016) Timing matters: open-loop stimulation does not improve overnight consolidation of word pairs in humans. Eur J Neurosci 44:2357-2368. 